\title{
Ethnomedicinal Resources of the Indigenous People's (IP) Groups in the SOCSARGEN Region
}

\author{
Maria Luisa Non-Cabrera ${ }^{13}$, Angem Librando-Descallar ${ }^{1}$, \\ Christine Dawn G. Obemio ${ }^{1}$, Tres Tinna B. Martin-dela Cruz ${ }^{1}$, Rhumer Lañojan ${ }^{2}$ \\ ${ }^{1}$ Faculty, Mindanao State University, General Santos City Science Department \\ ${ }^{2}$ Faculty, Mindanao State University, General Santos City College of Education Training Department \\ ${ }^{3}$ Corresponding author, mailbox.mlnon@gmail.com
}

Received: October 6, 2017; Accepted: March 13, 2018

\begin{abstract}
An ethnomedicinal study was conducted to document the medicinal plants used, the manner of preparation and application in the treatment of ailments and the consensus in the use of medicinal plants to treat various ailments among four (4) indigenous people's groups, the Obo in Lake Sebu, South Cotabato, T'boli in Maitum, Sarangani Province, and Blaan and Tagakaolo in General Santos City. The ethnobotanical interview was conducted using open-ended semi-structured questionnaires among identified traditional healers and elders as well as the household in the community. A total of 136 plants species, mostly herbs (47\%), have been recorded with the corresponding conditions or illnesses in which they are believed to exert a therapeutic effect. Plant parts utilized for traditional medicine of the 4 IP groups include leaves (38\%), roots (25\%) and bark/stem (16\%). Decoction (54\%) and direct consumption (27\%) applied via oral route are the common traditional medicine preparation. The IP communities in the SOCSARGEN Region have a rich pharmacopeia of traditional medicinal plants that persist through time despite the availability and convenience offered by modern medicine.
\end{abstract}

Keywords: ethnobotany, traditional medicine, T'boli, Obo, Blaan, Tagakaolo, informant consensus factor $(I C F)$

The universal desire to live a disease-free life provided means for the utilization of natural resources as therapeutic solutions. Throughout history, the use of traditional medicinal plants for combating various ailments can be confirmed by available references presenting rituals and spiritual healing practices of different indigenous groups in the country. Since time immemorial, traditional medicine played a vital role in the healthcare and maintenance of indigenous communities.

Mendoza (2009) reported that alternative medicine is popular in the country and at present indigenous sources such as plant and animal parts are still the most important supply and sometimes the only source of therapeutics for indigenous people. In her study on "Mainstreaming Traditional Health Knowledge," Palaganas et al. (2001) discussed that traditional healing practices, to include the use of medicinal plants, are favored over consultations and check-ups from medical profession- als. Preference to traditional healing may be due to inaccessibility of healthcare/medical services to IP communities and their low economic status, conditions common in most IP communities.

Traditional use of biological sourced medicinal resources plays an important role in modern drug discovery. Various researches conducted in India, Europe, Asia and South American countries utilized the ethnomedical information of plants as reference to indicate the presence of biologically active constituents (Union Internationale, 1993; Pfeiffer and Butz, 2005; Garcia et al., 2010, Sher et al., 2010, Sivaperumal et al., 2010). Studies showed that therapeutic claims of medicinal folklores were in many cases proven to be scientifically valid. These folk or traditional medicines served as 'leads' or clues to the discovery of modern medicines. Currently, a large number of medicinal plants has found their way as raw materials of the modern biopharmaceutical industry. These and 
more have led to the evolution and establishment of new and recently well-recognized scientific endeavor, the ethnopharmacology which brings together traditional and scientific medicinal/ pharmaceutical principles (Heinrich and Gibbons, 2001, Gilani, \& Atta-ur-Rahman, 2005; Pieters and Vlietinck, 2005, Nishijima et al., 2009).

At present, the use of traditional medicine has tremendously expanded and gained nationwide acceptance and popularity considering the expensive western medical treatment which most Filipinos could not afford. The approval of Republic Act 8423 (R.A. 8423) otherwise known as the Traditional and Alternative Medicine Act (TAMA) of 1997 that promotes and advocates the use of traditional medicine nationwide bolstered the popularity and quantum recognition in the mainstream of traditional medicine (Mendoza, 2009).

SOCSARGEN region is home to diverse biological resources that includes some endemic species in its Key Biodiversity Areas (KBA) namely; Daguma Range, Mt. Matutum Protected Landscape and Mt. Busa. These natural landscapes are also the original domain of different ethnic groups such as T'boli, Blaan, Tagakaolo, Obo and Manobo before the settlement of migrants in the majority of the lowland areas of the region (PGO-South Cotabato, 2013; PSA, 2012). The researchers selected the landscape as the site of the study being the home of various Indigenous People (IP) Groups (Conservation International Philippines et al.,
2007)

According to de la Cruz and Gorospe (2006), IPs are very close to nature that the very essence of their cultural heritage depends on their interaction with it. Highly integral in IP's culture is traditional healing practices utilizing flora or fauna combined and in most cases with associated rituals. Each IP has its own distinct cultural and traditional practices although they may sometimes slightly resemble each other. For sure these are shaped by their natural environment and their interaction with varied neighbouring ethnic groups, migrants, the Moros and other tribal people. This study sought to conduct ethnopharmacological resource assessment in major IP communities of SOCSARGEN region. Such undertaking hopes to widen knowledge based on current IP traditional pharmaceutical/medicinal resources and its utilization in the region.

\section{Materials and Methods}

\section{Study Area}

SOCSARGEN region situated in the southernmost part of Mindanao is composed of South Cotabato, Sarangani Province, and General Santos City (Figure 1). It is a home to the diverse group of indigenous communities who have been wandering the area for hundreds of years (Peralta, 2003). Indigenous groups from this region, T'boli, Obo, Blaan, and Tagakaolo were selected for eth-

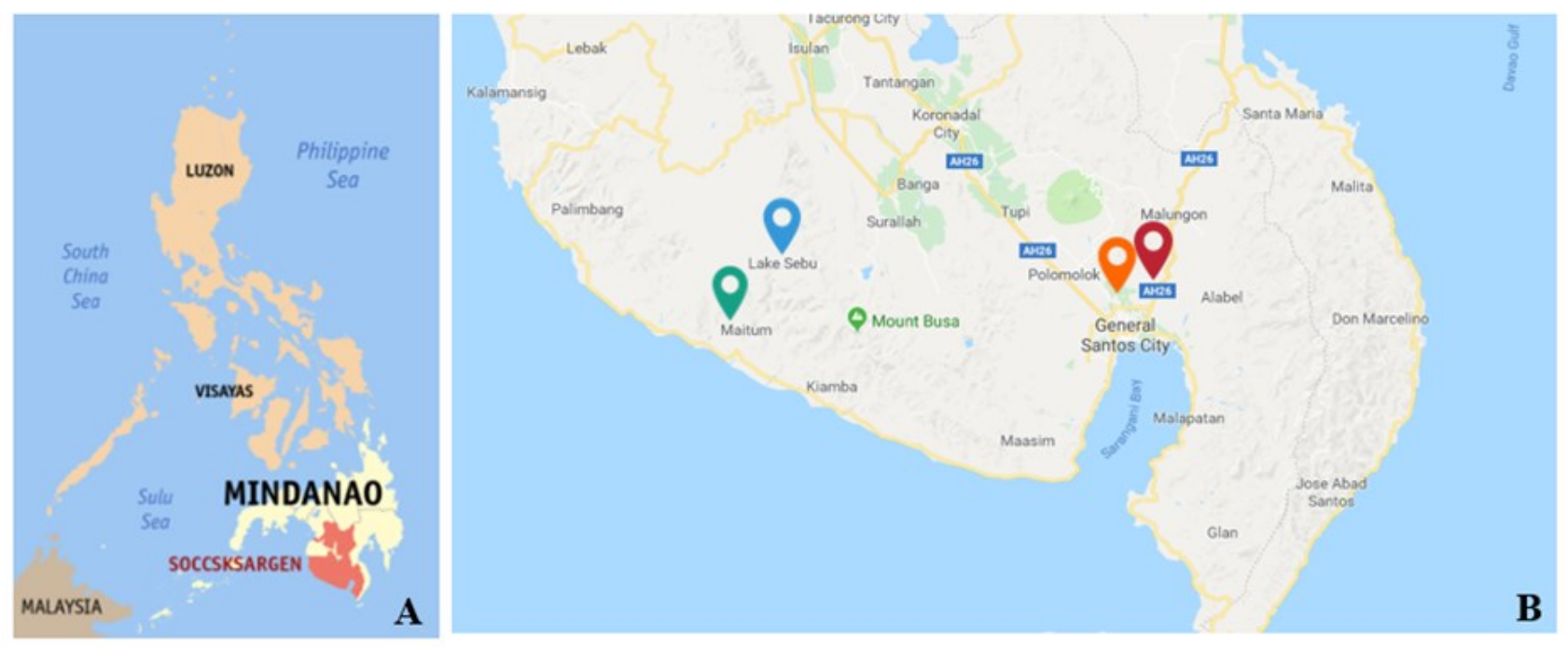

Figure 1. Satellite map showing the location of A) SOCCSKSARGEN Region in Mindanao, and B) the IP communities marked as study sites: 9 Sitio Sansapan Brgy. Upper Labay, General Santos City, Blaan community (N $06^{\circ} 17.654$ E $125^{\circ} 11.448,165-616$ masl); S Sitio Bunga, Brgy Conel, General Santos City (N 06 14.09 .72 E $125^{\circ} 09.55 .82,145-194$ masl); 9 Sitio Upo, Brgy. Lanao, Maitum, Sarangani Province, T’boli community (N 06 ${ }^{\circ} 07.015$ E $124^{\circ} 27.450,108-678$ masl); Sitio Lambila, Brgy Lamfugon, Lake Sebu, South Cotabato, Obo community (N 06 16.814 E $124^{\circ} 38.898,503$ -619 masl). Sources: https://en.wikipedia.org/wiki/SOCCSKSARGEN, www.Googleearth.com 
nopharmacological data collection.

Rugged hills and dense vegetation surround the study sites. Sitio Sansapan Brgy. Upper Labay, General Santos City of the Blaan community, is particularly located at $\mathrm{N} 06^{\circ} 17.654$ E $125^{\circ} 11.448$ with an elevation of 165-616 masl; Sitio Bunga, Brgy. Conel situated N 06 ${ }^{\circ} 14.09 .72$ E $125^{\circ} 09.55 .82$ at $145-194$ masl is the location of a small community of Tagakaolo; Sitio Upo, Brgy. Lanao, Maitum, Sarangani Province for the T'boli community located $\mathrm{N} 06^{\circ} 07.015$ E $124^{\circ} 27.450$ with an elevation of 108-678 masl; and Sitio Lambila, Brgy Lamfugon, Lake Sebu, South Cotabato for the Obo community located at $\mathrm{N} 06^{\circ}$ 16.814 E $124^{\circ} 38.898$ with an elevation of 503619 masl. All these cultural communities were observed to rely on crop agriculture as their main source of livelihood and are dependent on rainfall to water their ground.

Permits, Courtesy Calls, and Ocular Inspection

Before the conduct of the study, the research team acquired the necessary permits from the Department of Environment and Natural Resources, and the Indigenous People's Council in the respective municipalities/city selected for the study. Courtesy calls and ocular inspection to the identified study sites-municipal and barangay level were made. Meetings with officials of Barangay and Municipalities to present the project and address some critical issues were also conducted.

\section{Ethno-Pharmacological Survey}

A reconnaissance survey was conducted to every potential study site before the actual field study. The survey was done with the purpose of identifying contact person from every chosen community to facilitate entry to study sites. It also included the identification of local guides and interpreter who assisted the research team in the conduct of the study on identified study sites. Local guides possessing sufficient to excellent indigenous knowledge of the biological resources of the study site were selected for the field study.

Research permit from the Tribal Council of each community was secured before data gathering. Gratuitous Permit for the collection of sample specimen was also secured from DENR-12 office.

Documentation of the medicinal plants used by the indigenous people was carried out among purposively selected respondents. A total of 98 respondents from the four communities were interviewed using semi-structured interview based on the prepared questionnaire. Local IPs who have knowledge or are known users of traditional floral medicine were chosen as respondents with the help of local official, tribal leader and or local elderly people. A particular preference was given to traditional healers of the community. Eight tribal healers were interviewed, three (3) from each Blaan and T'boli communities and two (2) from each Tagakaolo and Obo communities. Age, sex, occupation and educational background of the respondents were noted. These factors as identified by Pfeiffer (2005) as including geographical origin, residence, ethnicity, and religion influences ethnobotanical knowledge. Selection of the actual sites for specimen collection was decided upon by the local guides and recommendations of the locals who are familiar with the area.

Prior to the interview, free and prior consent was secured from each respondent. The interview was done in Bisaya and Ilonggo dialects while an interpreter was sought for respondents who only speak their native language. Consent to record the process, such as photo documentation and video or audio recordings was also asked. None of the key informants objected to the documentation.

Obo, T'boli, Tagakaolo and Blaan field guides who also happened to be healers walked along the sampling sites with the researchers while being interviewed. Plants with medicinal value encountered along the way were identified with their medicinal use. Preparation and application of plants as medicine were described and demonstrated by the healers to the researchers. A Blaan healer even performed a simulated healing procedure that includes performance of a ritual. The actual preparation of medicine was documented in video and photographs. Respondents not identified as healers were interviewed through a house to house visit which was then followed by a survey of ethnomedicinal plants available in their backyards.

The plants used by the people in the community were photographed in their natural habitat and collected whenever possible. Several plants were collected from respondents' backyard but most were obtained from the forest. Mature plant specimens collected were pressed as voucher specimens and kept in the Nutraceautical Laboratory College of Natural Sciences and Mathematics, MSU, General Santos City. Observations on the morphological features and habitats of each medicinal species were noted during the field study to facilitate the complete taxonomic identification and classification of plant specimens. Online and in print sources were used for the identification of plants (Castro, 2006; CO's Digital Flora, 2011; 
JHRS, Vol. 1, 2018

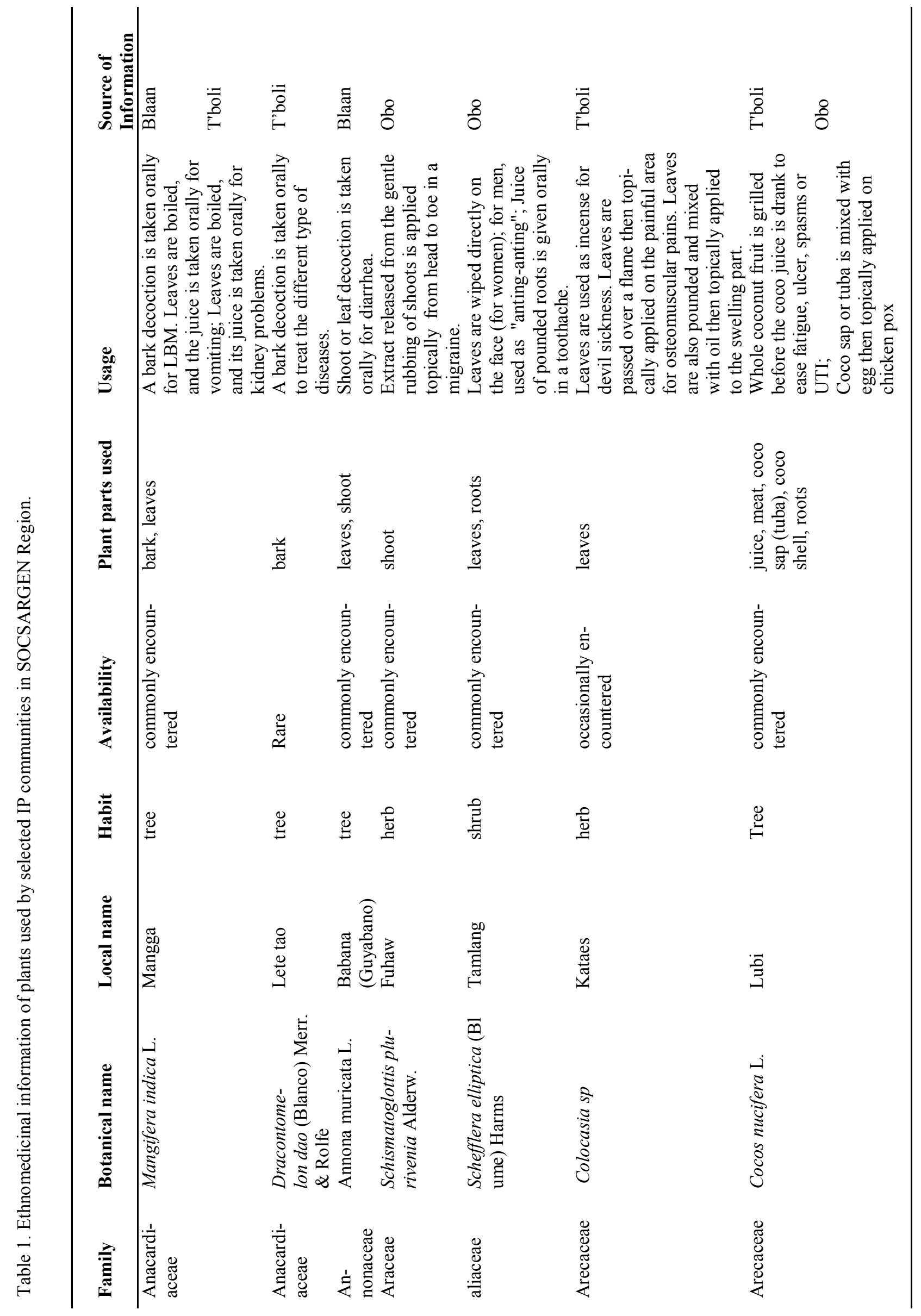




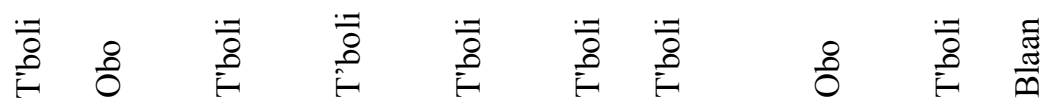

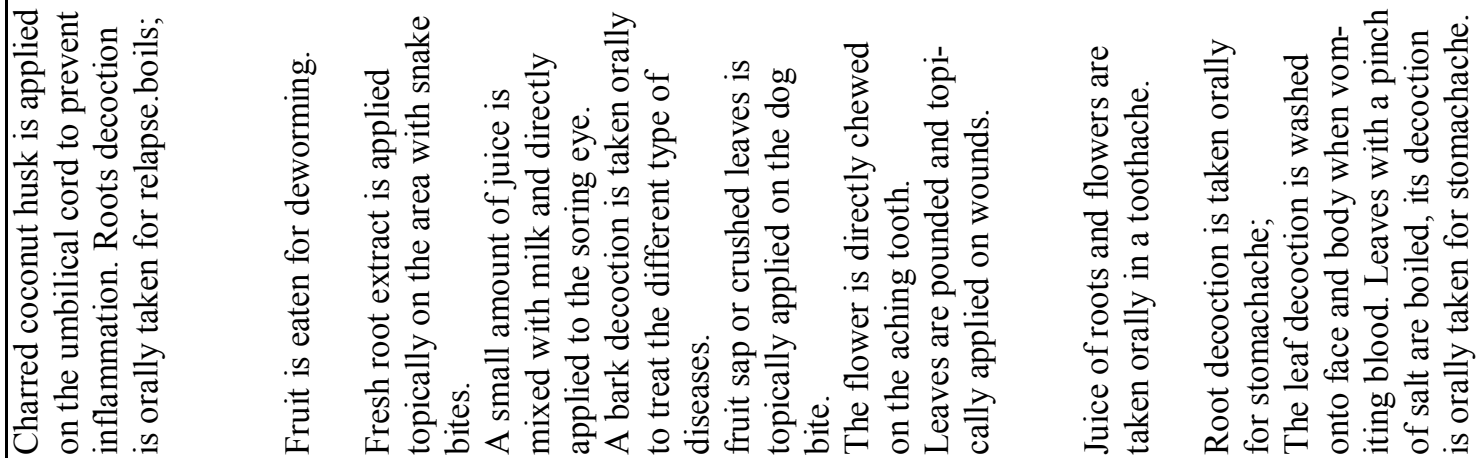

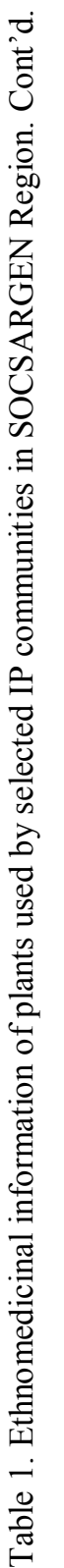

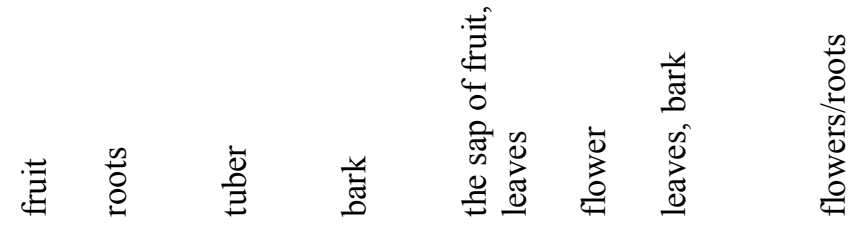

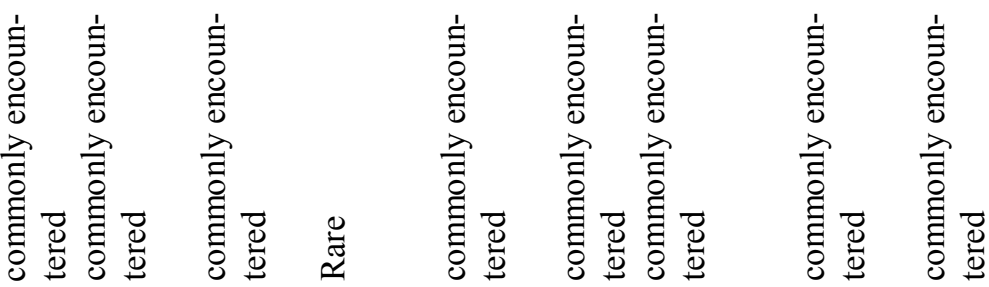

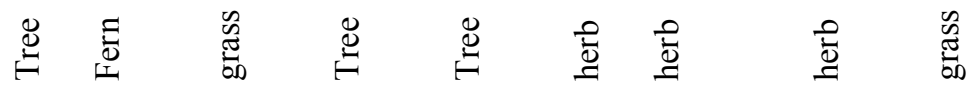

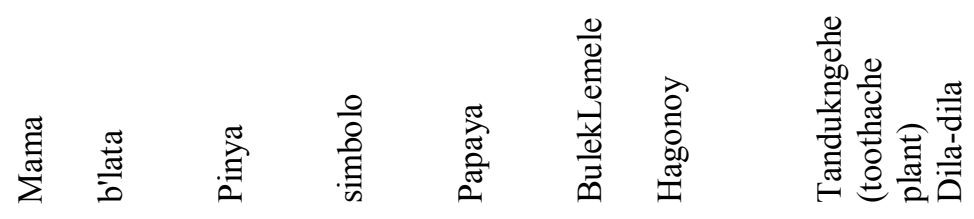

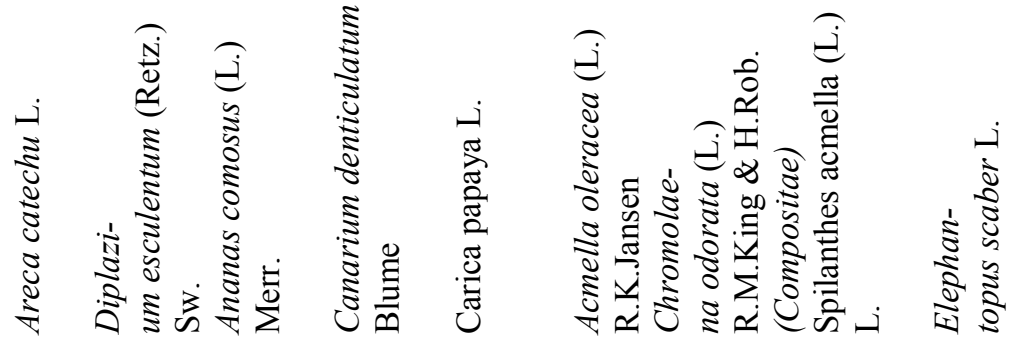

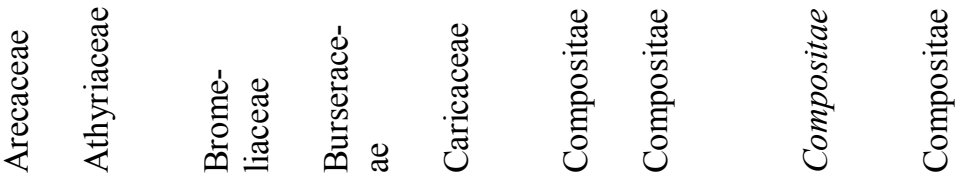




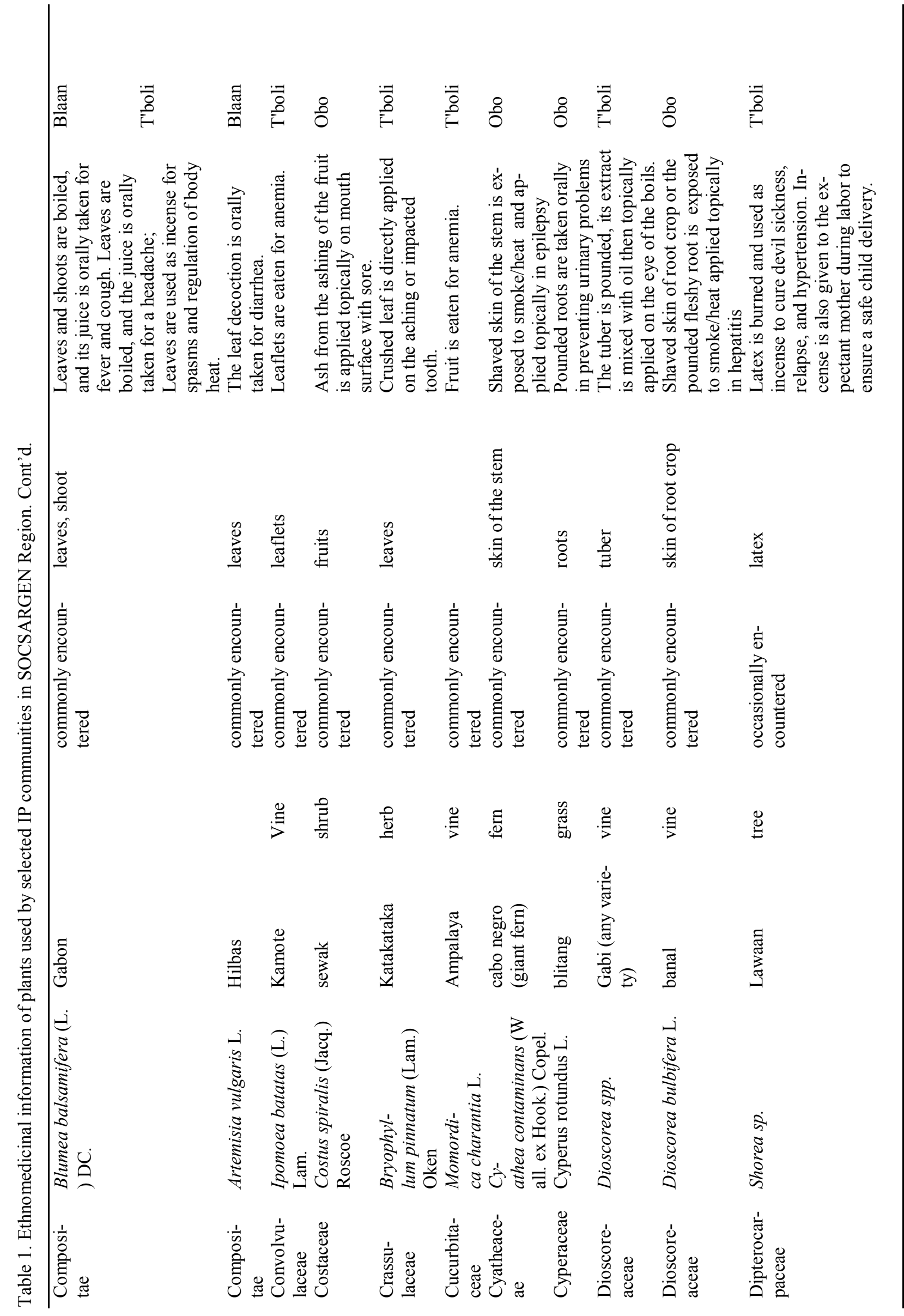




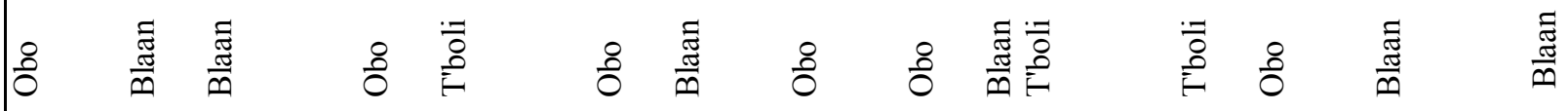

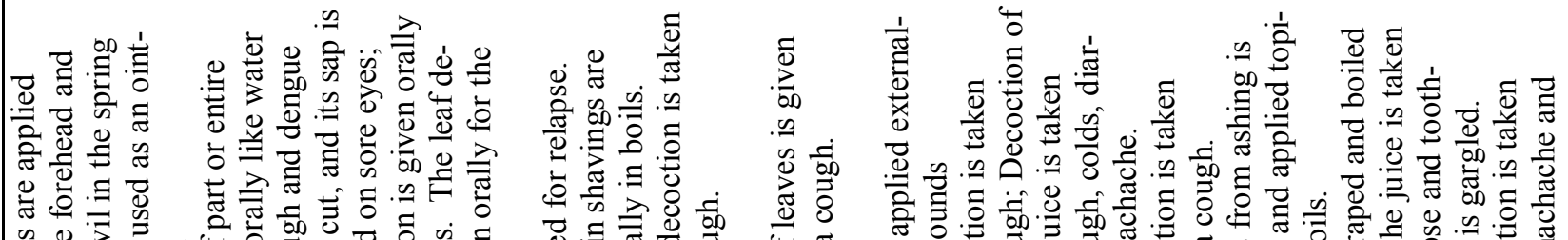
乡

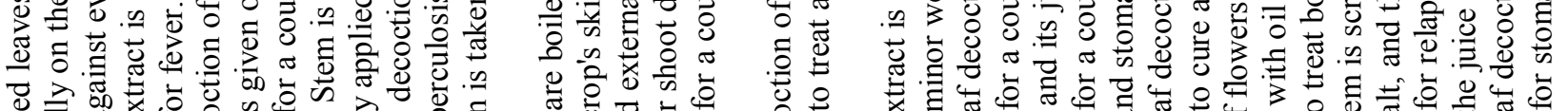

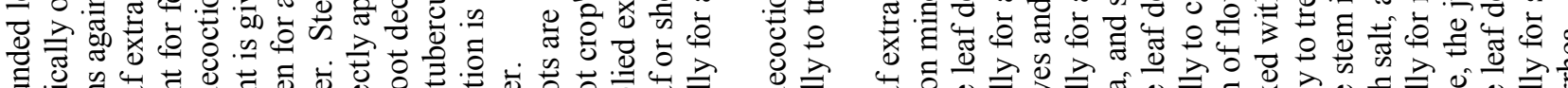

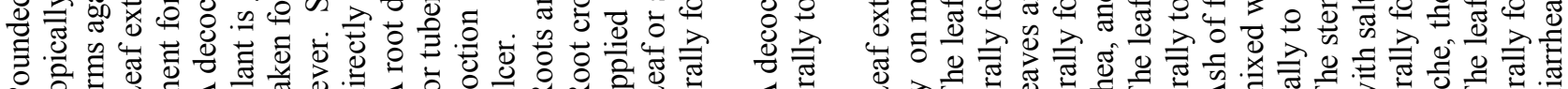

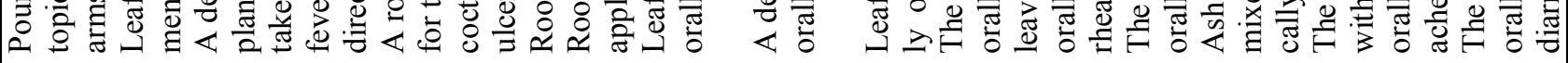

苛

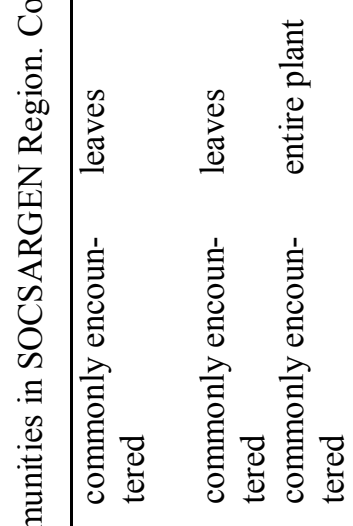

恶

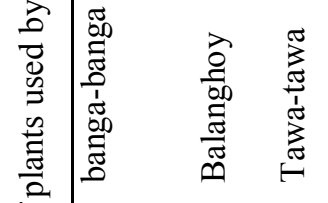

范

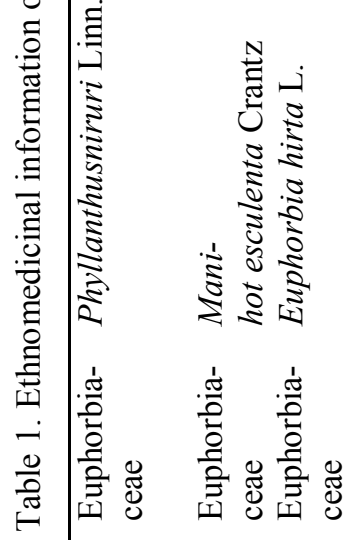

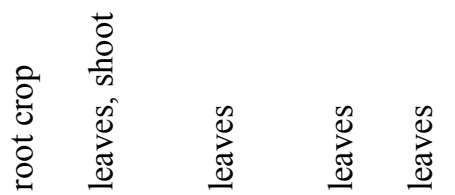

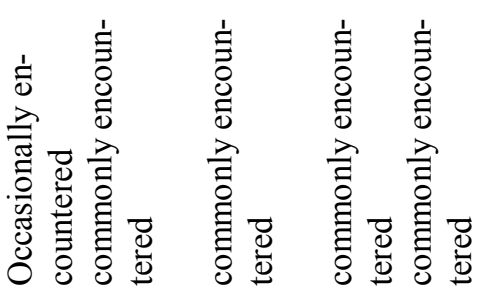

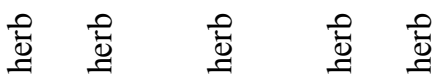

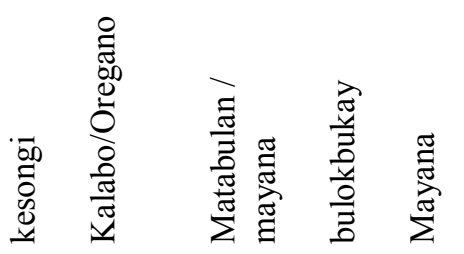

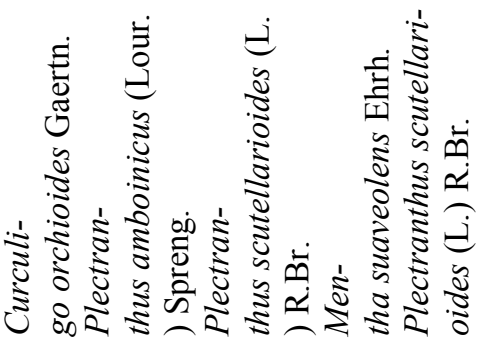

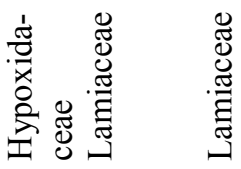

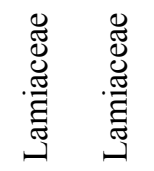

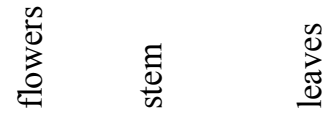

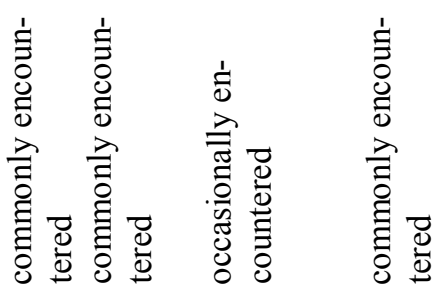

$\stackrel{\Xi}{\Xi} \stackrel{\Xi}{\Xi}$

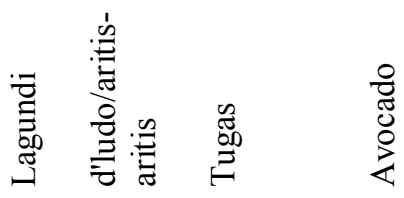




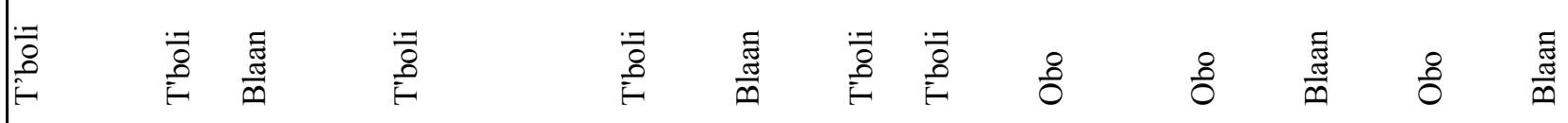

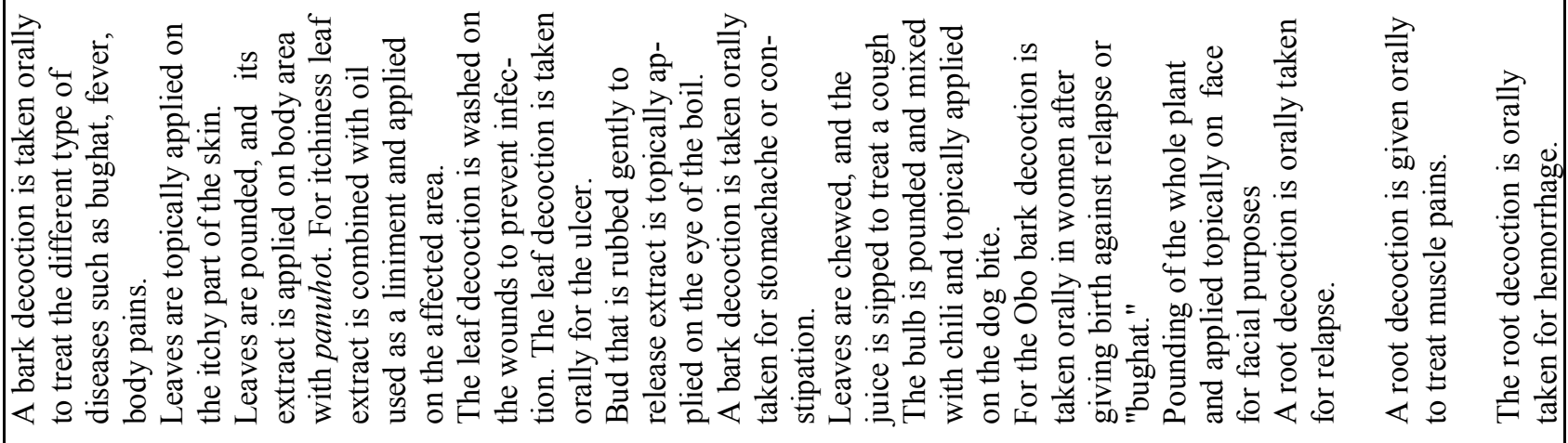

葡

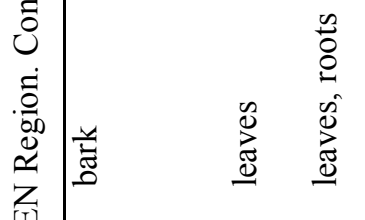

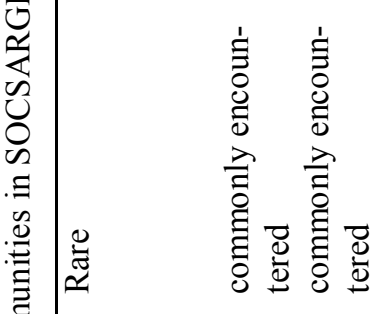

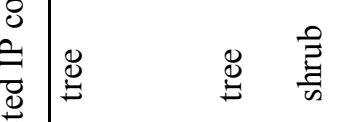

这

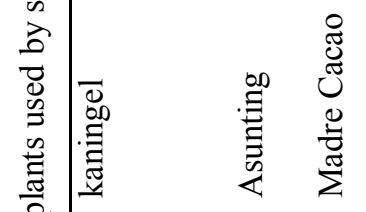

늘

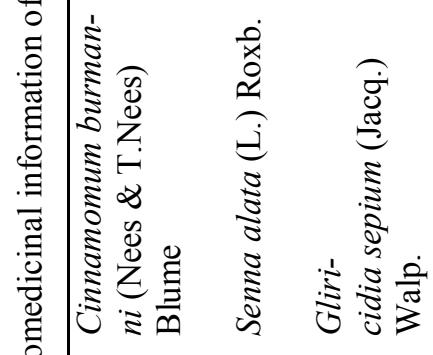

害

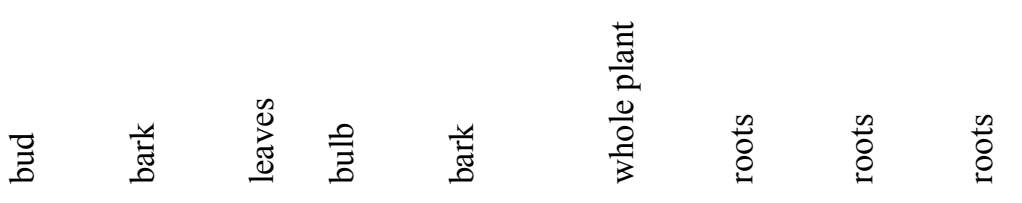

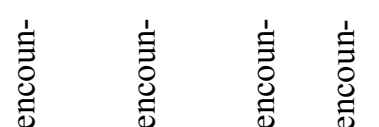

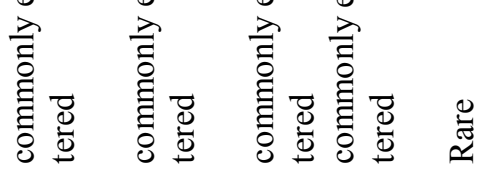

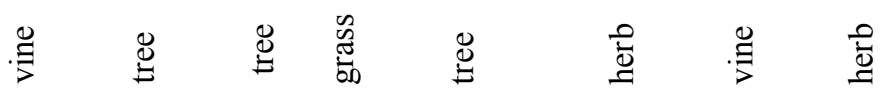

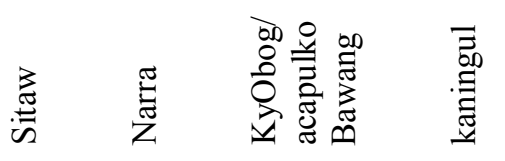

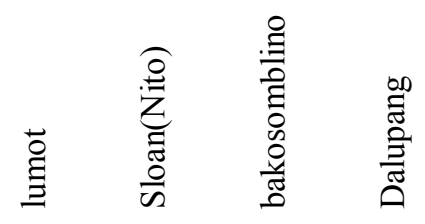

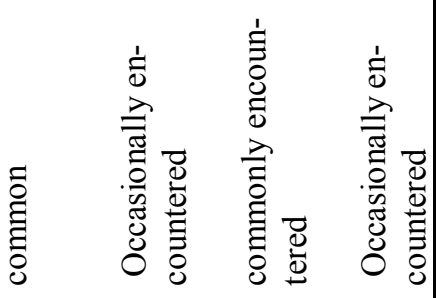

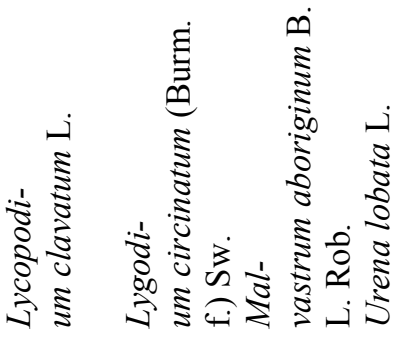

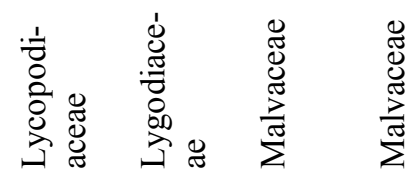




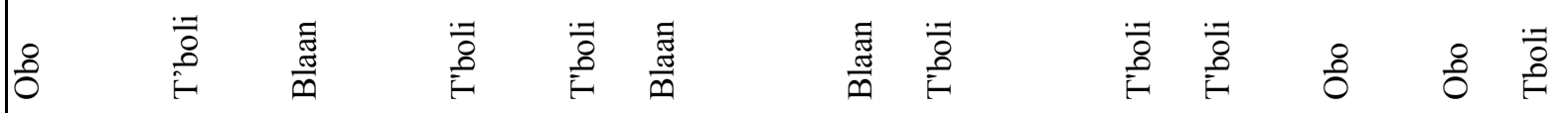

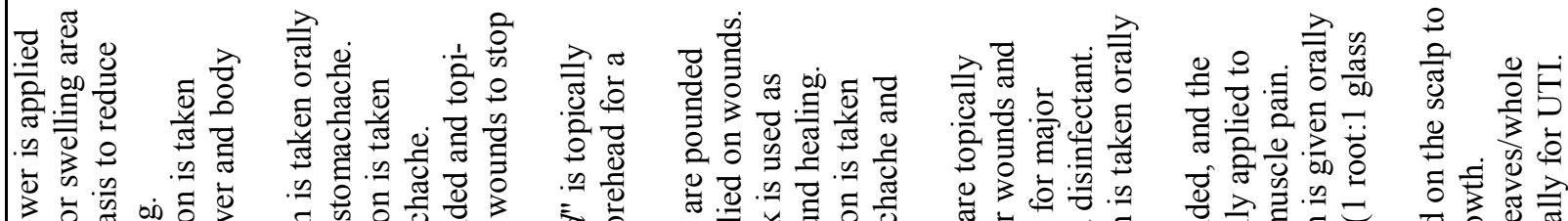

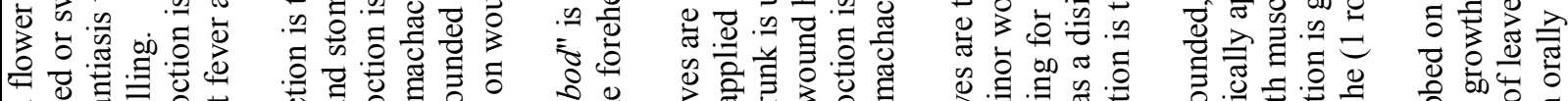
品击范

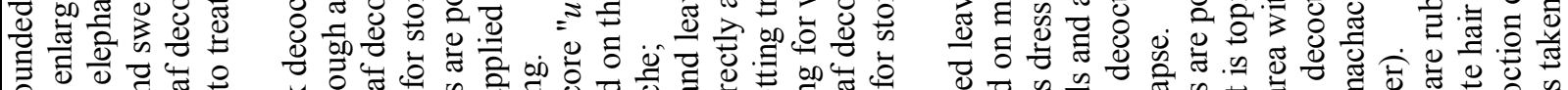

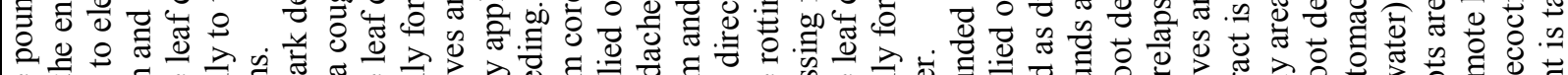

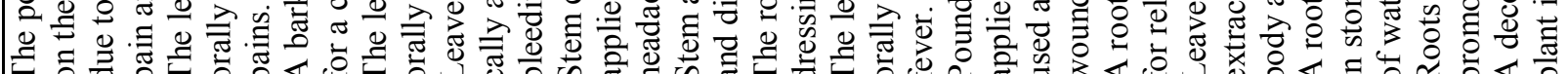

.

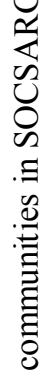

当

党

잉

.

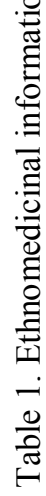

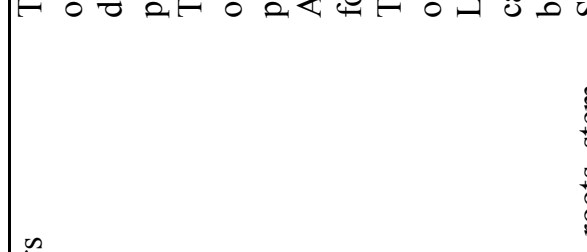

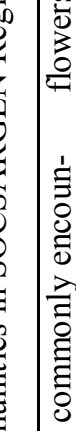

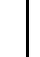

?

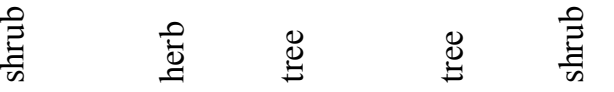

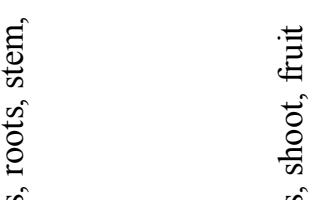

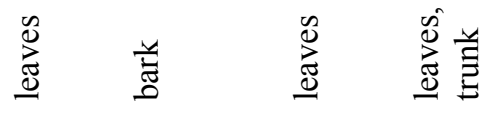

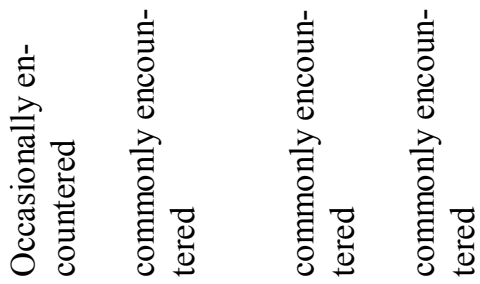

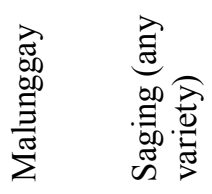

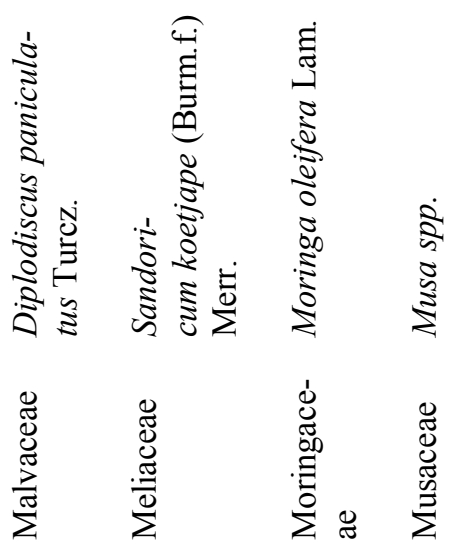

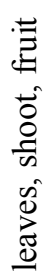

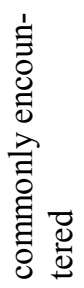

蛋

苞

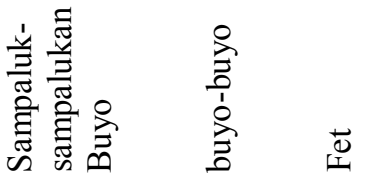

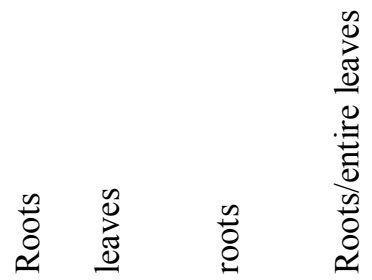

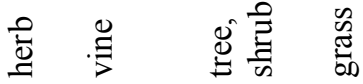
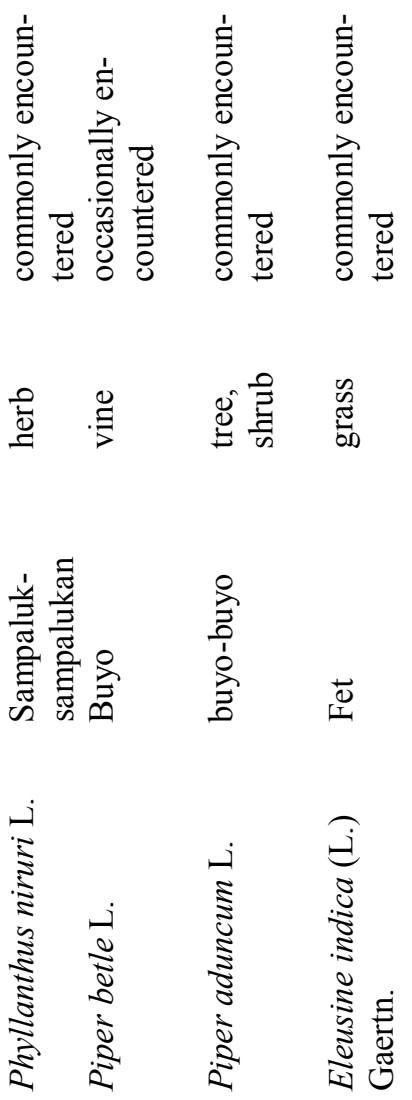

芯

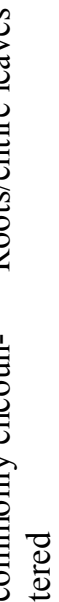

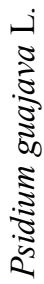

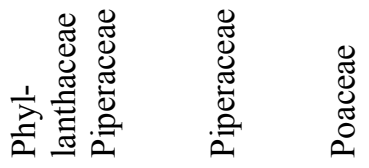




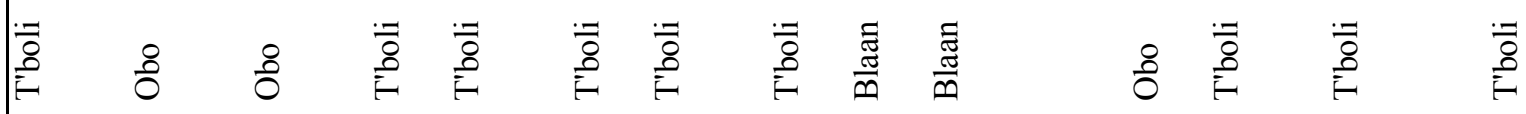

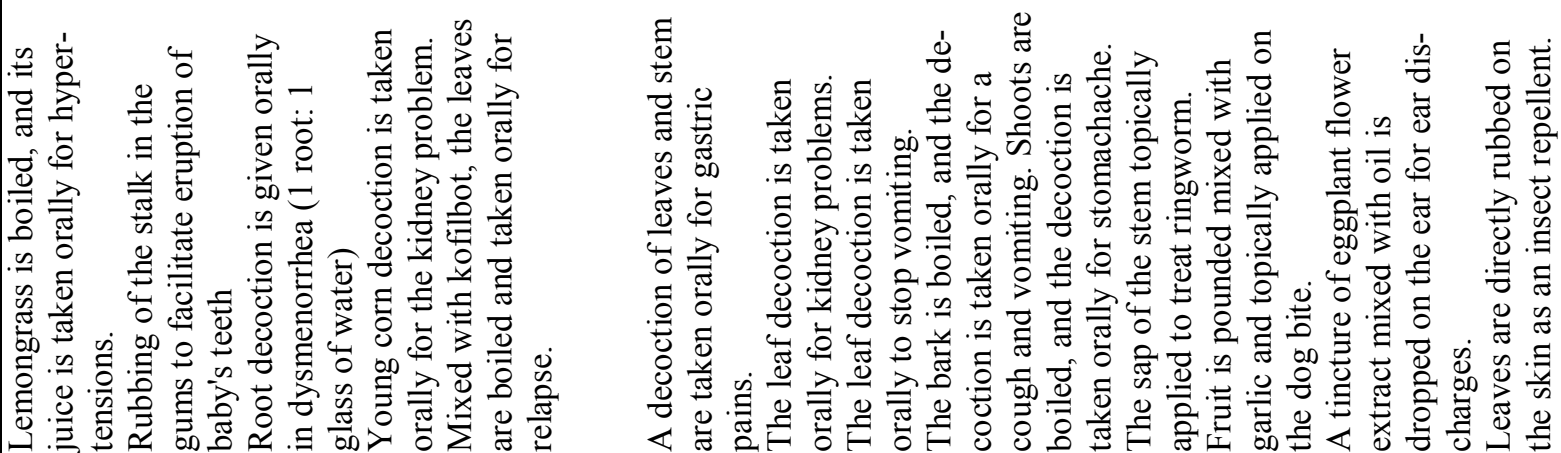

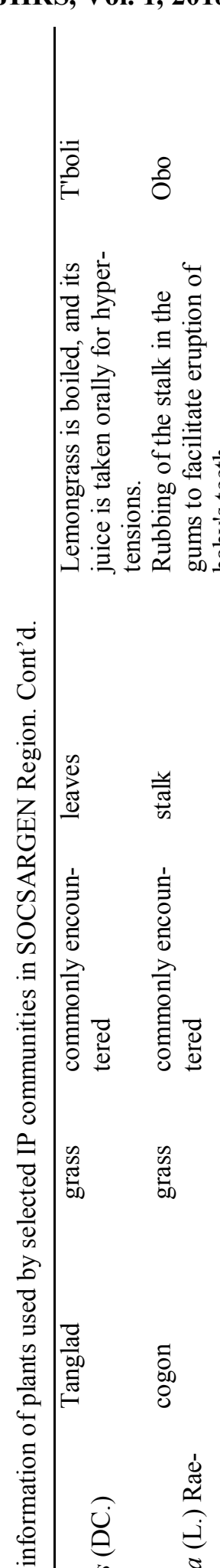

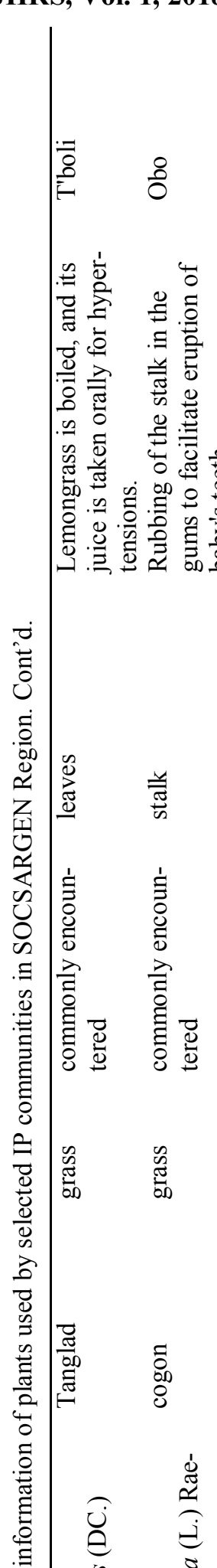

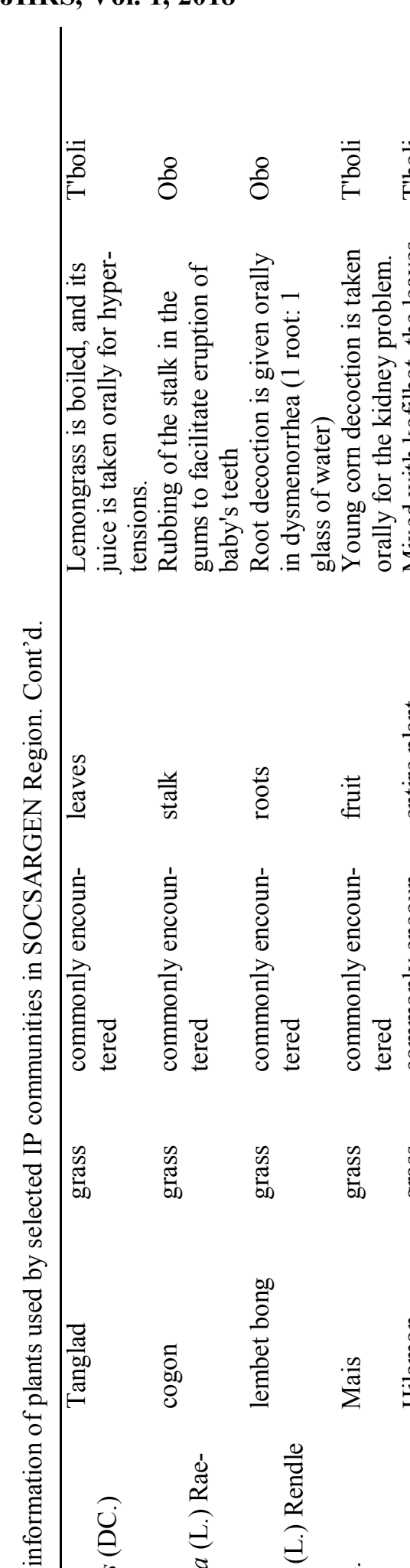

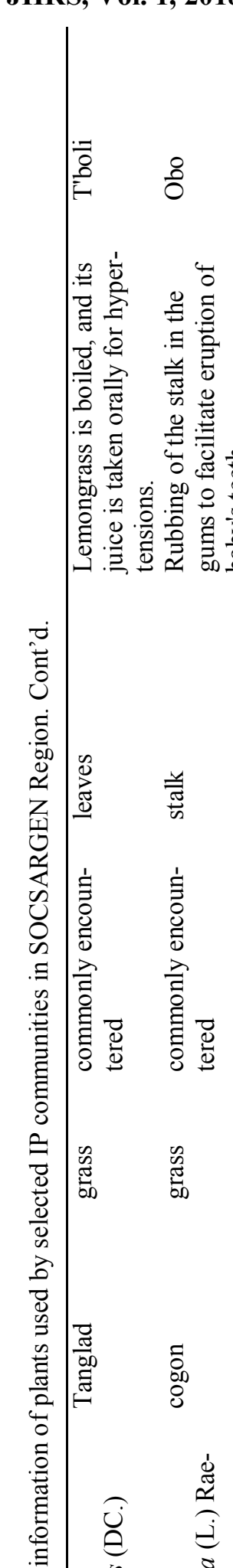

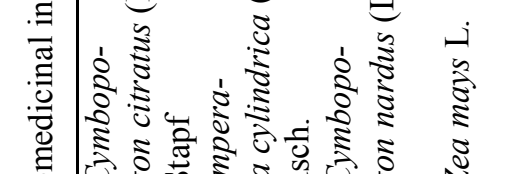

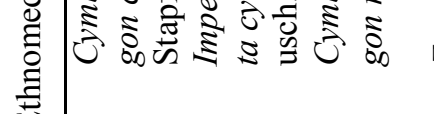

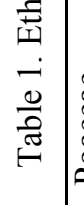

苛 苛

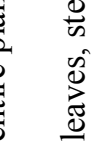

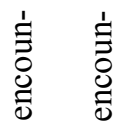

畜
产

竞

言

啇

(5)

s

总高

莺: 击

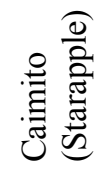

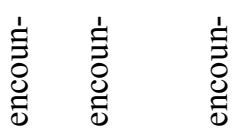

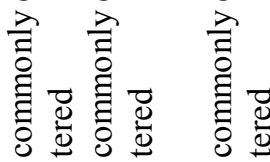

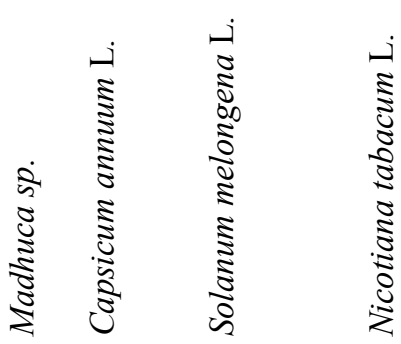




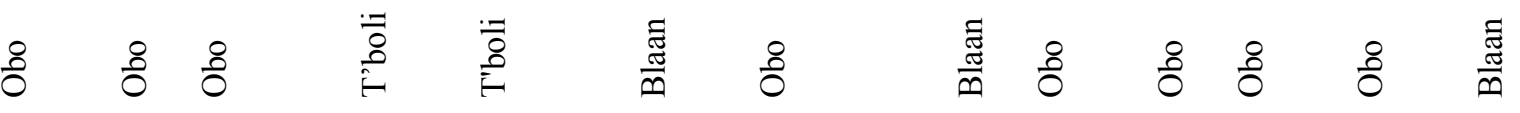

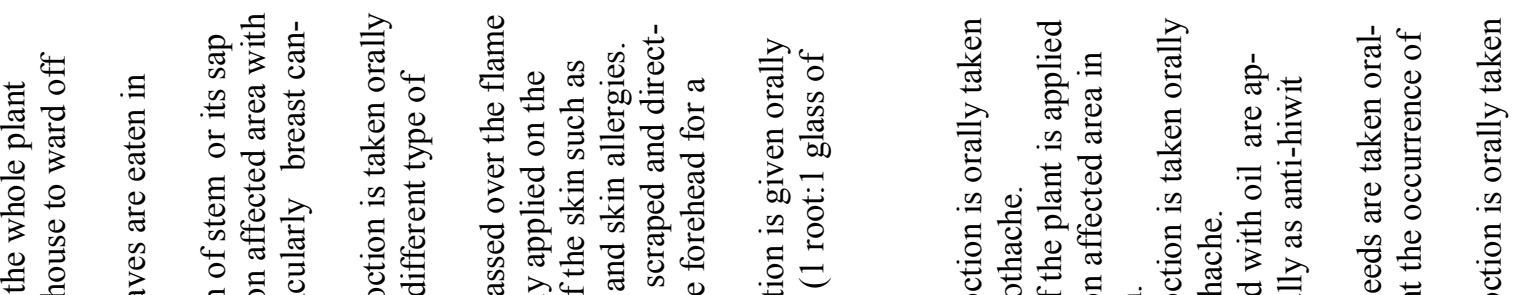

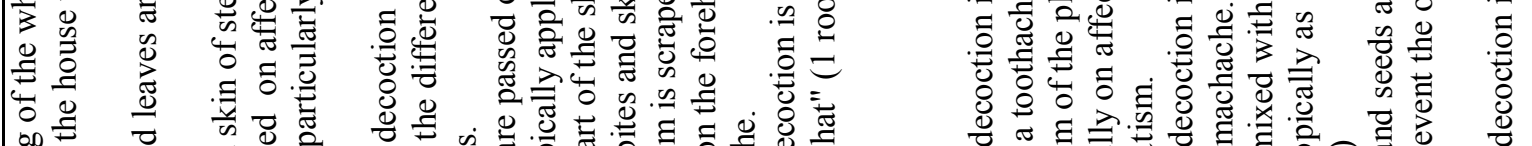

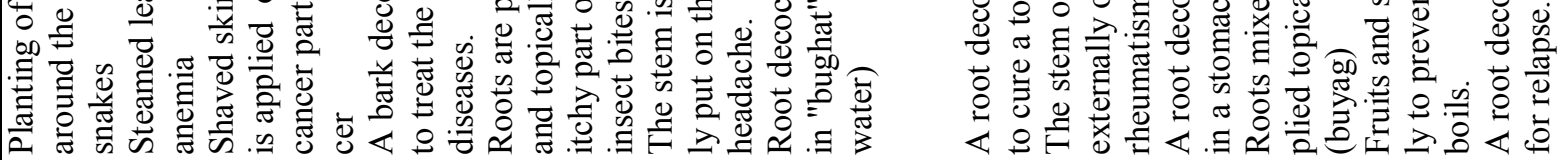

苛

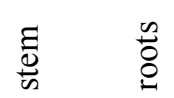

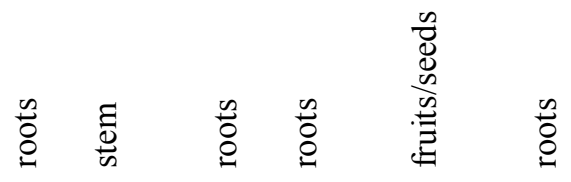<smiles>C1CCCC1</smiles>

.

त्र<smiles>CCCCCCCC</smiles><smiles>CC(C)C</smiles>

章

言

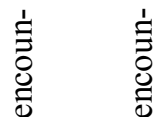

苛总<smiles>[CH]1C=CC1</smiles>

总

촐

촐

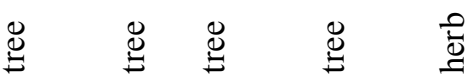

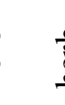
長

$\stackrel{\Xi}{\Xi}$

월

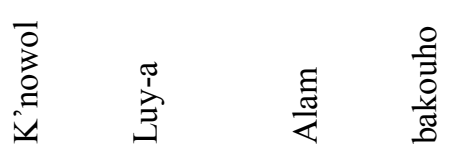

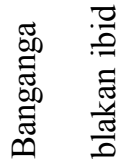

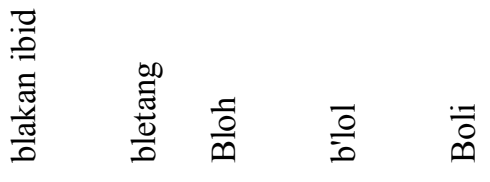




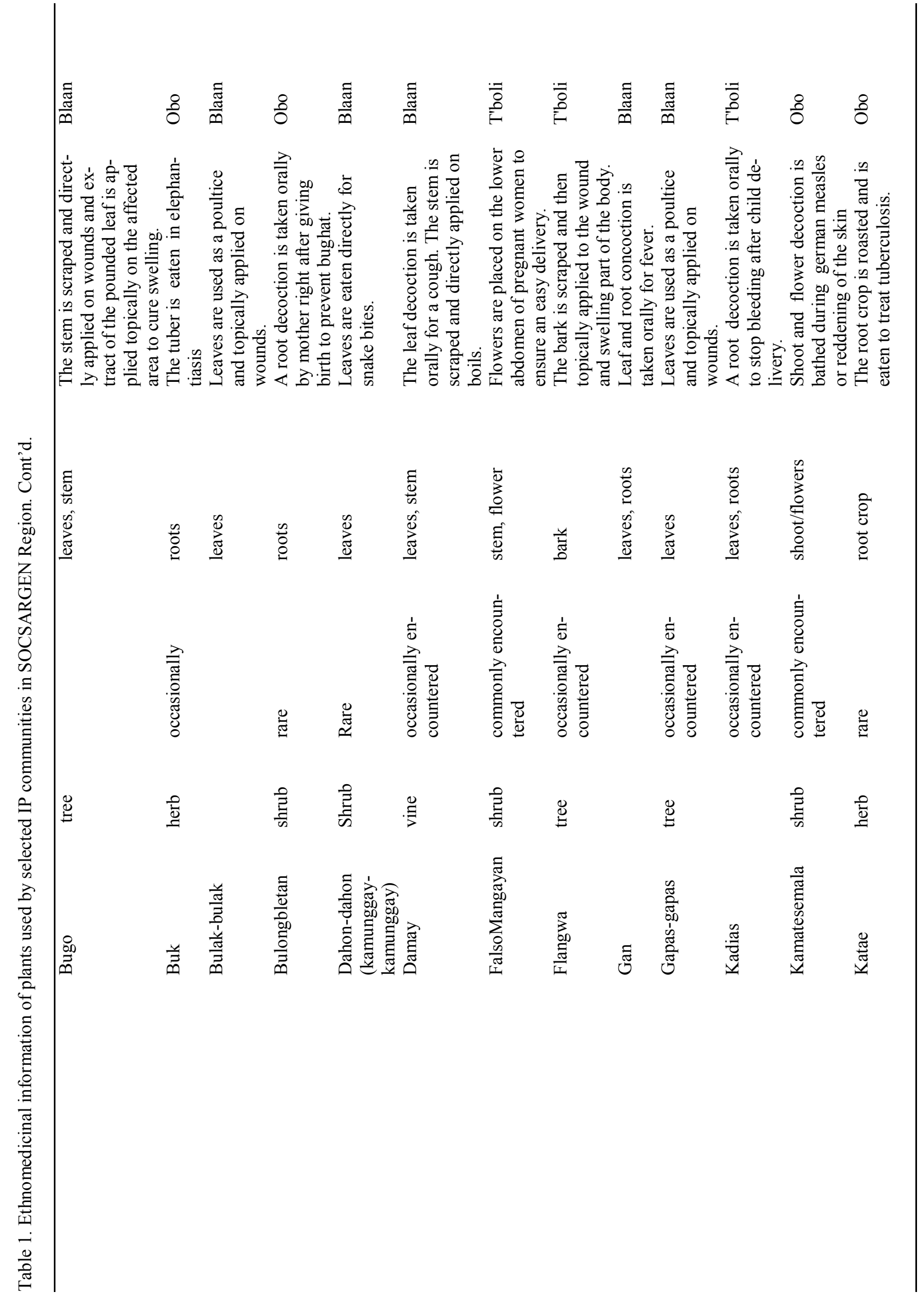




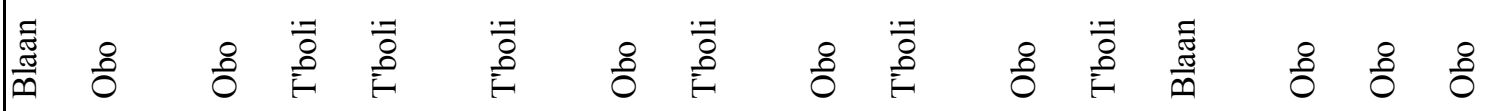

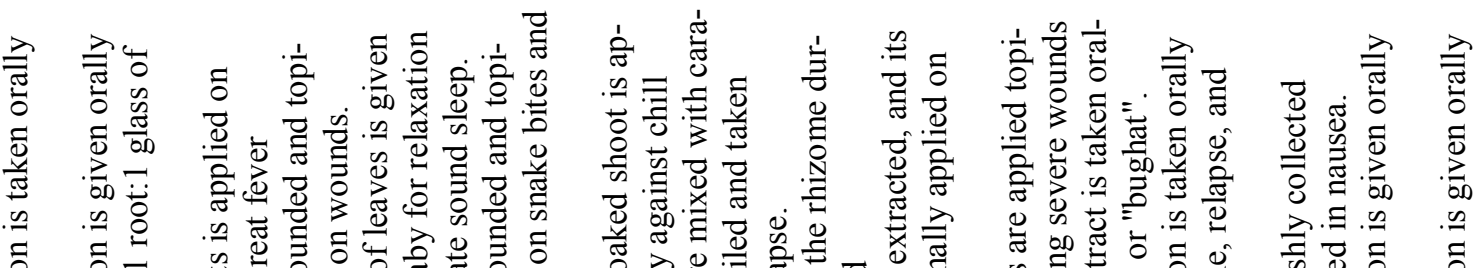

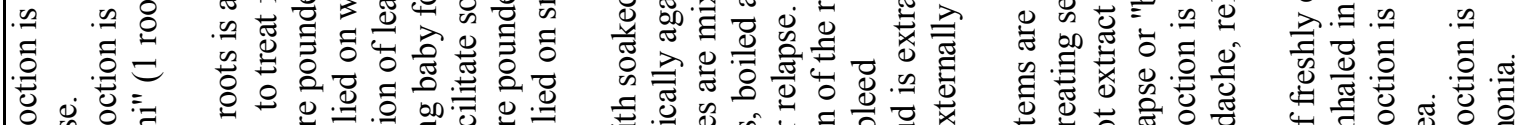

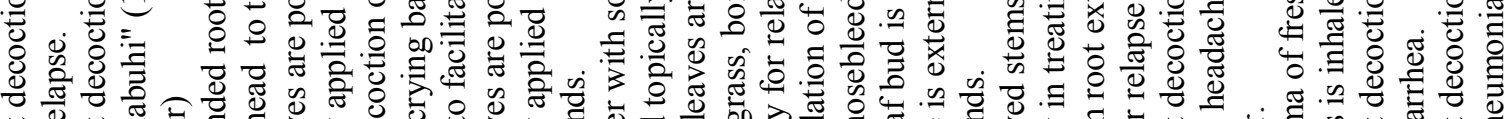

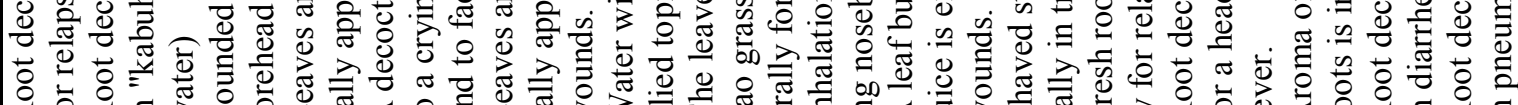

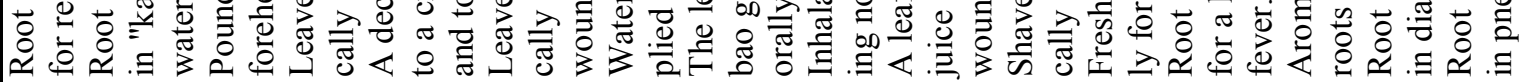

苛

.

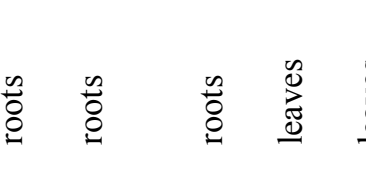

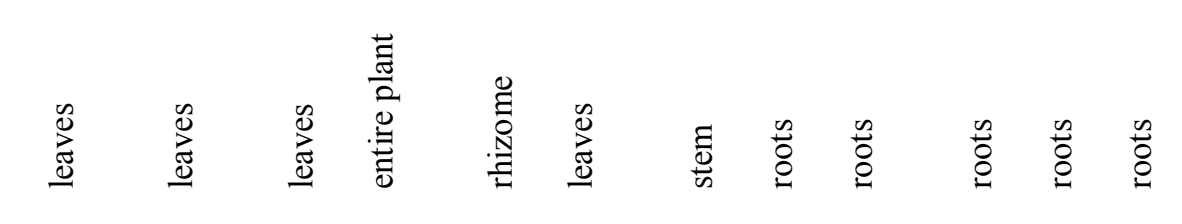

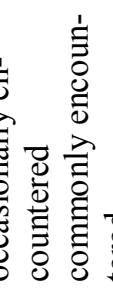
एँ

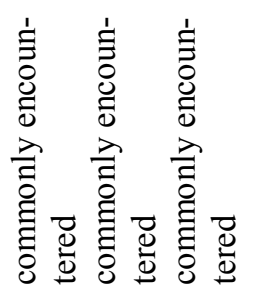

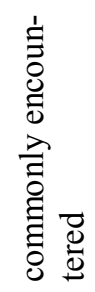

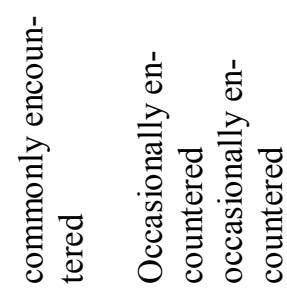

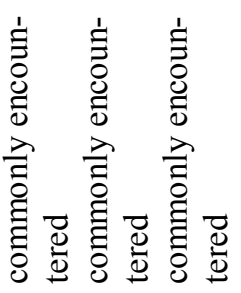

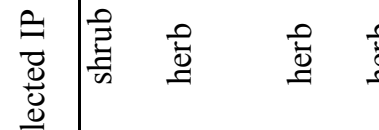

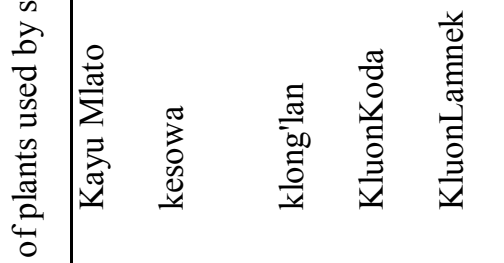
量

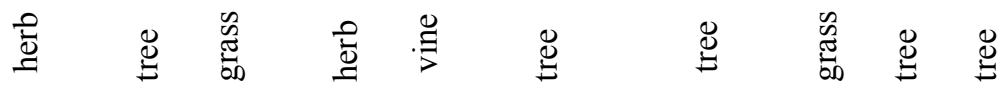

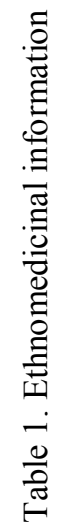

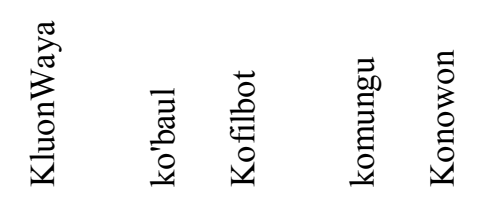

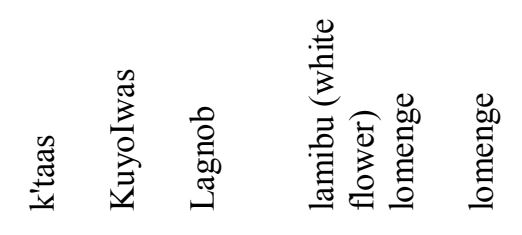




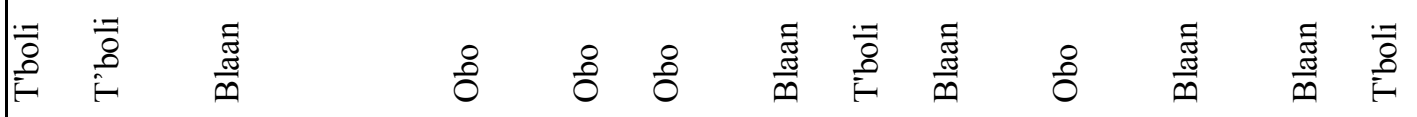

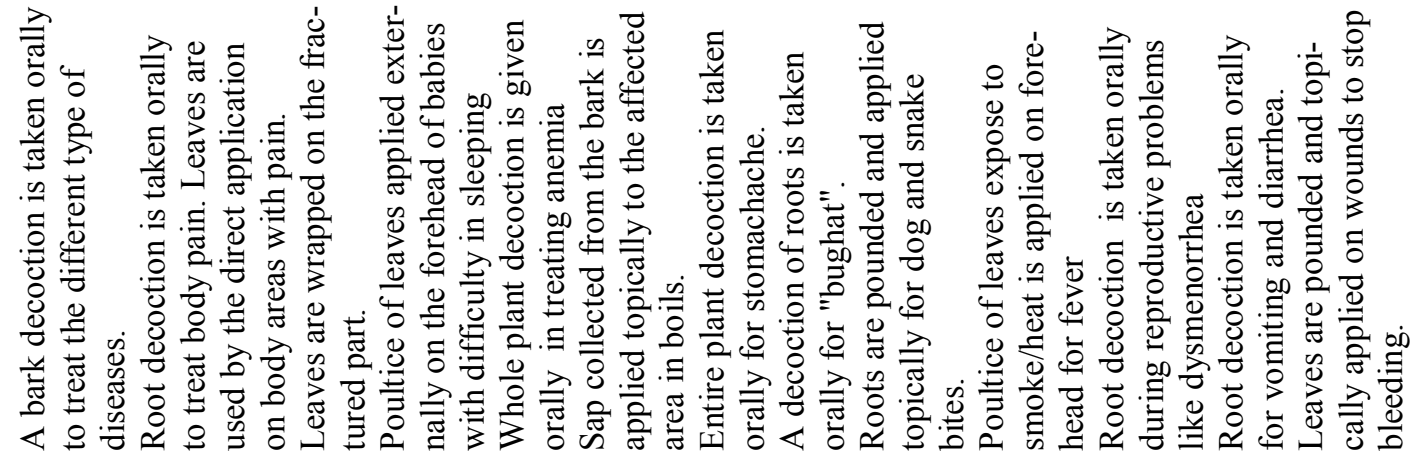


JHRS, Vol. 1, 2018

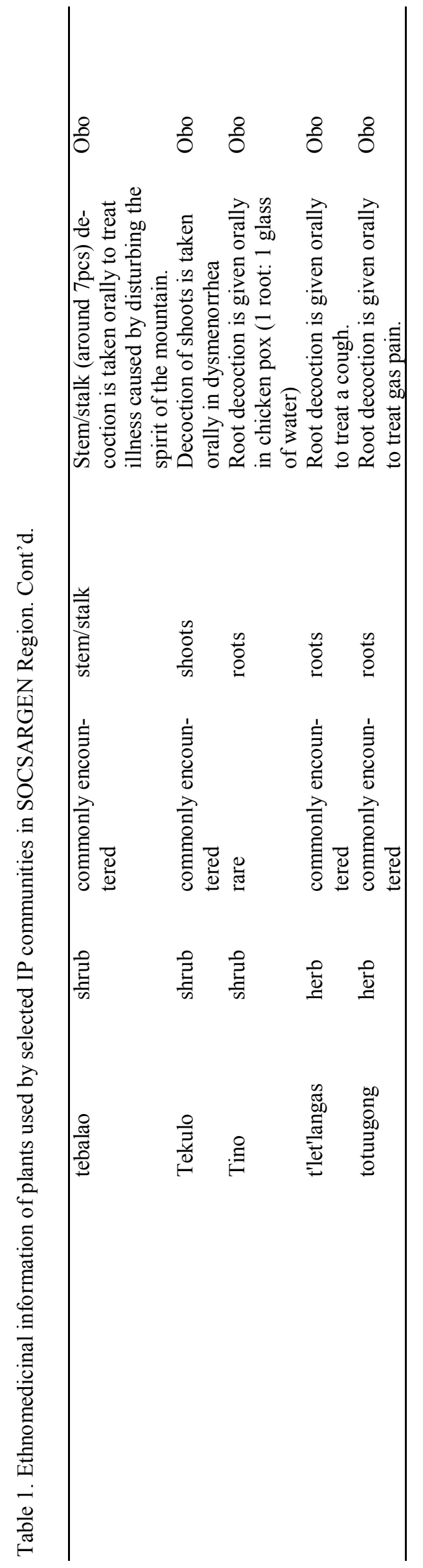

光

를

윰

包 $\frac{5}{0}$

टे हे

0
0
0
0
0
0

ธิธิธี

突泀

จे

: 5

ज

४导

可路

突吉

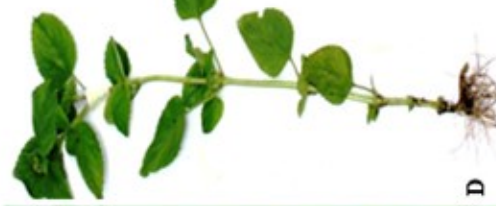

00

宅. on

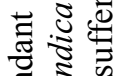

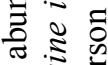

on 동

8 四

00

宅

해월

응

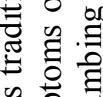

朦寻

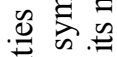

苛

寻.

8 일

宅

ลे 3

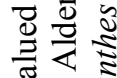

ㄱำ

दे को

엘

象

흘

to 0

원

है हैํㅀ

沱

ำิ

兽岕 
Plant List, 2013) with several plant specimens confirmed by a plant taxonomist from the National Museum. Prevailing diseases in the area were classified into sixteen categories based but with some modifications from the classification made by Ferreira, et al., (2009). The categories were assigned in numbers, each presenting a disease relating to a particular organ of the body.

\section{Results and Discussions}

\section{Ethnobotanical Resources of IP groups}

Medicinal plants play an important role in providing knowledge to the researchers in the field of ethnobotany and ethnopharmacology. The observations of present study showed that traditional medicine plays a significant role among the members of the Obo, T'boli, Blaan and Tagakaolo. The tribes' cultural practice of treating diseases always involves utilization of medicinal plants.

One hundred thirty-six (136) plants were recorded based on interviews of IP traditional healers and users, seventy-eight (78) of which were identified at species level. These plants were used to treat 44 types of various ailments and diseases sorted out into 16 categories. Species are distributed to 39 families dominated by Lamiaceae (18\%), Poaceae (18\%), Compositae (15\%), Leguminosae $(13 \%)$, Solanaceae $(13 \%)$, and Malvaceae (10\%). Table 1 records these floral species including the ethnomedicinal information. Sample of the plants that are abundant and commonly utilized for treating common health problems are Zingiber officinale Roscoe, Schismatoglottis plurivenia Alderw, Eleusine indica (L.) Gaertn, Mentha suaveolens Ehrh, and Spilanthes acmella (L.) L. Photos with the information of medicinal uses are shown in Figure 2.

Based on respondents' assessment of medicinal resources, $66 \%$ were commonly encountered, $0.8 \%$ was moderate, $9 \%$ occasionally encountered, $10.5 \%$ rare and $13.5 \%$ of species with no available information. These values express the availability of the medicinal plants. Commonly encountered plant species or those abundant are normally utilized by the respondents for convenience. Moreover, healers sometimes explore the deep forest areas just to get medicinal plants to heal his suffering patient as discussed during the interview. Most of the diseases reported can be attributed to some external factors such as the weather conditions, from the immediate environment, their way of life, as well as the homeostatic and physiological disparities.
The range of gastrointestinal problems and internal parasite infestation, genito-urinary problems, cuts and wounds, and illnesses associated with child delivery such as relapse, bleeding, and muscle weakness are problems which have rich floral based pharmacopeia. Most medicinal plant species were used to treat only one disease with a few that can cure more than one disease. Particular plants of interest for their multiple uses are kaningel, (Cinnamomum burmanni (Nees \& T.Nees) Blume) and tawa-tawa (Euphorbia hirta L.).

Tawa-tawa (Euphorbia hirta L.) is reported to cure tuberculosis, sore eyes and malaria. Kaningel (Cinnamomum burmanni (Nees \& T.Nees) Blume) also showed a wide range of medicinal uses particularly on women's health problems. The multi-therapeutic ability implies that these plant species have the capability of curing wide range of conditions demonstrating their usefulness as direct source of traditional medicine.

Majority of the medicinal plants listed in Table 1 are used singly with only $3 \%$ used in combination with other plants or animal by-products such as milk. Example of medicine preparation using a combination of plants are the shredded kaningag bark and dalupang (Urena lobata L) bark wrapped in gabi (Dioscorea spp), fuhaw (Schismatoglottis plurivenia Alderw) or any available broad leaf inserted inside a bamboo and cooked on fire. The juice extracted in this process is then taken orally to cure genito-urinary problems among women.

\section{Method of Preparation, Plant Part/s Used, Ap-} plication, Growth Forms

The type of organ/part used (stem including modified stems rhizome and tuber, leaves, bark, roots, shoot, latex/sap, seed, fruit, flower, or whole plant) in the preparation were identified; and methods of preparation of treatments (poultice or passing over flame, infusion, decoction, tincture, ash application, burned, eaten raw/juice is extracted, cooked without seasoning) and route of application (oral, nasal, external/topical skin), hitting the body, bath/wash, exposure to smoke/heat) were also documented (Figure 3). Source of collection was also determined to evaluate the location of the species, whether it was domesticated or can be sourced only in the forest. Information generated may be useful in assessing the ecological status of the medicinal plants.

Among the different plant parts, the leaves were most frequently used for the treatment of diseases having the highest percentage (38\%) followed by roots $(25 \%)$ and stem $(16 \%)$. Figure $4 \mathrm{~A}$ 

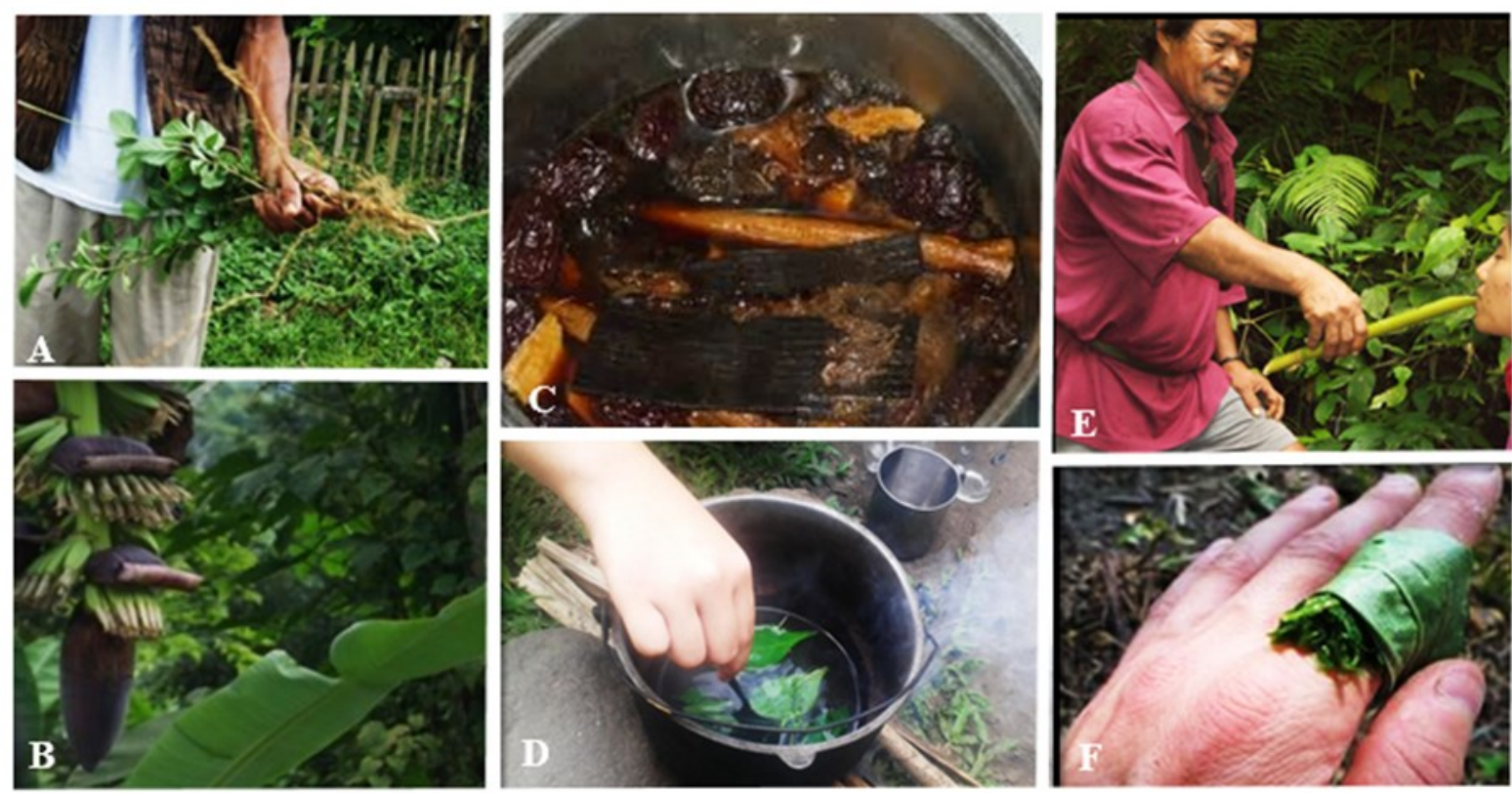

Figure 3. The various ways of preparation and application of traditional medicine, with roots, stems (A), and leaves (B) as the common plant parts used. A typical preparation is through decoction $(\mathrm{C} \& \mathrm{D})$ which may be taken orally $(\mathrm{E})$ or used in bathing. For topical application (F), plant parts are commonly pounded to release the sap.

shows the preferred parts of plants used in treating the ailments in the community. In the study of Leffers (2003), who presented and described 238 plant species occurring in the eastern Namibia, as well as in the studies of Kumar et. al. (2007) and Panghal et. al. (2010) a wide range of different plants and plant parts were used with plant roots playing a significantly important role. Findings of this study, however, showed roots to be only the second popular choice in plant part utilization.

The analysis of growth forms shows that, of the total medicinal plants recorded from the study sites, herbs were highly represented having a percentage of $43 \%$ followed by shrubs, trees, grasses, ferns, and vines (Figure 4B). Higher use of herbs for medicinal purposes is attributed to perceived effectiveness in the treatment of ailments and diseases. As compared to the other growth forms, natural abundance and availability made it all the more the most preferred growth form of plant.

In Figure 4C, it was evident that the most common method of medicine preparation was decoction (34\%), followed by direct consumption, crushing/pounding, poultice, ash application, shaving, steaming, rubbing, and direct inhalation. Boiling leaves and roots in water often result in extraction of oils, volatile organic compounds and other chemical substances that brought about the effectiveness of the treatment in curing ailments (Capasso, et al., 2003; Azmir, et al , 2013).
Figure 4D shows that $50.98 \%$ of the medicines were administered orally, $43.14 \%$ topically while exposure to heat/smoke and hitting the body accounts for the remaining $5.88 \%$. This means that treatments were usually swallowed or taken in to guarantee the efficacy of the medicine.

\section{Informant Consensus in the Use of Medicinal Plants}

Informant consensus factor (ICF) was determined per category. ICF is used to measure the consensus among respondents from the four communities regarding the information they have given in the use of floral species in the treatment of a certain group of ailments (Table 2). The value describes the degree of agreement of people in the community in the treatment of diseases. Value of 1 means high consensus of plants utilized for treatment. Tabuti, et al. (2012) interpreted that a high ICF value means that the community is confident in the choice of plants for medicine, while a low ICF value means that the community is still experimenting and that the treatment may not be sufficient.

A low consensus was observed among informants in Brgy. Upper Labay, General Santos City with regards to the plants they utilized to treat diseases. Dermatological problems, and infection categories, however, yielded an ICF value of 1 . In addressing dermatological problems, madre de cacao (Gliricidia sepium (Jacq.) Walp) is used as an ointment to treat itchiness and infection, while 
A

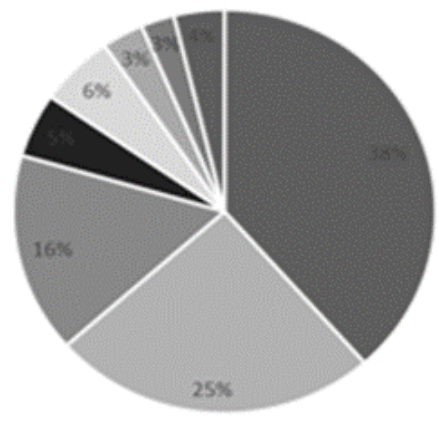

- Leaves $\quad$ = Roots

- Flowers an Fruits

= Whole plant $=$ others

C

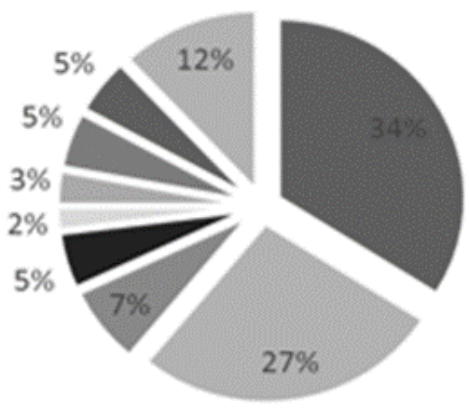

- Bark/Stem

- Sap

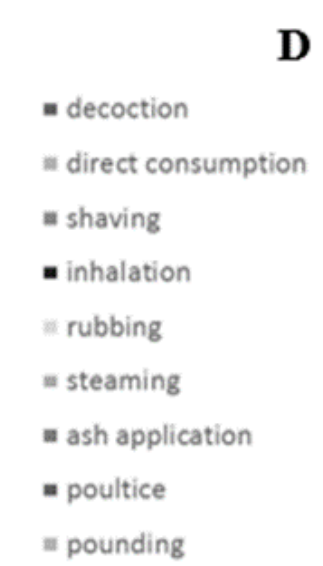

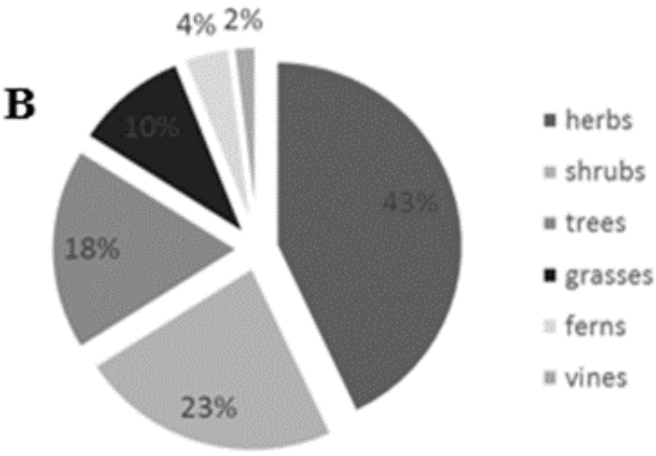

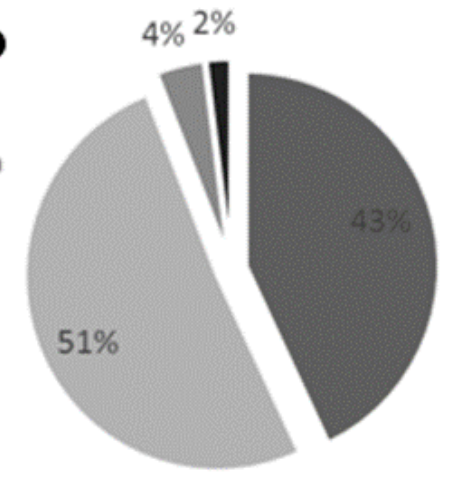

m topical

w oral

= exposure to heat/smoke

m hitting the body

Figure 4. Preparation and use of traditional medicinal plants. (A) Preference of tribal healers in terms of plant parts used; (B) composition of medicinal plants based on habit; (C) preferences of traditional healers for route of administration; and (D) mode of medicine preparation for traditional healing.

decoction of tawa-tawa (Euphorbia hirta L.) is popularly used to treat dengue.

With the current effective health program of Barangay Upper Labay and easy access to their constituents to medical needs (Brgy. Upper Labay Profile Report, 2012) most residents prefer western medicine to treat illnesses. However, if the sickness is worst, has no progress while on treatment or requires immediate treatment as in the case of snake bites, and the treatment using conventional western medication is expensive patients and their family resort to traditional medicinal way of treatment. Eye and ear related category, antidote-related category, and cancer obtained the highest ICF Value of 1 in the Blaan community of Upper Labay. This means that all informants in the community use similar selection of plants to treat a disease. Tawa-tawa (Euphorbia hirta L.) is used to treat sore eyes, b'lata as antidote for snake bites, and damay (Pipturus asper Wedd.) for treatment of cancer.
For Obo tribe, antidote has the highest ICF value. A range of plants are used as antidote for snake bites. Plants used as antidote may be available in the backyard while others are only found in the forest. However, not all plants were documented by the researchers as they are guarded secrets of some healers. The tribe believes that the effectiveness of the antidote will be lost if made known to others. The plant is typically chewed to extract the juice and is applied on bitten body part with the bark as the common plant part harvested.

Wound healing for severe and minor wounds obtained the next highest ICF with a value of 0.875 (Table 2). K'taas for severe wound and bulokbukay (Mentha suaveolens Ehrh.) for minor wounds, which are common plants in the community, are applied to the wound with a gentle pressure until bleeding stops or pain becomes manageable. The nervous system related problem followed by infectious and transmitted diseases category also obtained high consensus among respondents 
JHRS, Vol. 1, 2018

Table 2. Informant consensus in the use of medicinal plants among IP communities in SOCSARGEN region.

\begin{tabular}{|c|c|c|c|c|c|}
\hline \multirow{2}{*}{ Disease Category } & \multirow{2}{*}{ List of Disease } & \multicolumn{4}{|c|}{ ICF } \\
\hline & & Tboli & Blaan & Obo & Tagakaolo \\
\hline Antidotes & Rabies & -0.5 & 0 & 1 & 0.76 \\
\hline Cancer Related & & - & - & 1 & \\
\hline $\begin{array}{l}\text { Cardiovascular System Related } \\
\text { problem }\end{array}$ & High Blood Pressure, anemia & -0.5 & 0 & 0.666 & 0.566 \\
\hline Dermatological Problems & $\begin{array}{l}\text { Itchiness, allergies, boils, insect } \\
\text { repellant, ap-ap }\end{array}$ & 0.00 & 1 & 0.809 & 0.809 \\
\hline Devil Sickness & Barang & 0 & - & 0.857 & 0.867 \\
\hline Eye and Ear Related & Sore eyes, buog (ear infection) & 0 & 0 & 1 & - \\
\hline Fever and Headache & Fever, headache & - & 0 & 0.846 & - \\
\hline $\begin{array}{l}\text { Gastro-intestinal and internal para- } \\
\text { sites }\end{array}$ & $\begin{array}{l}\text { Gastric Illness, stomachache, diar- } \\
\text { rhea, deworming }\end{array}$ & 0.36 & -0.0833 & 0.818 & - \\
\hline $\begin{array}{l}\text { Genito-urinary system related prob- } \\
\text { lems }\end{array}$ & $\begin{array}{l}\text { Smooth delivery, bleeding after } \\
\text { childbirth, relapse, breast pain, }\end{array}$ & 0.3333 & 0 & 0.777 & - \\
\hline Infection & $\begin{array}{l}\text { Dengue, chicken pox, malaria, } \\
\text { leprosy }\end{array}$ & 0.2 & 1 & 0.8333 & - \\
\hline Musculoskeletal and Other Pains & $\begin{array}{l}\text { body pain/muscle pain, spasm, } \\
\text { toothache }\end{array}$ & 0.2 & 0 & 0.769 & 0.59 \\
\hline Neurologic disorders & Paralysis & 0 & - & 0.842 & 0.842 \\
\hline Respiratory Disease & Cough & 0.4 & 0.00 & 0.818 & 0.818 \\
\hline Weakness & Fainting, weakness & 0.16667 & -0.2 & -0.2 & 1 \\
\hline Wound Healing & $\begin{array}{l}\text { Wound bleeding, wound healing, } \\
\text { swelling }\end{array}$ & -0.09 & 0.22 & 0.875 & 0.880 \\
\hline Miscellaneous & $\begin{array}{l}\text { crying of baby, facilitate eruption } \\
\text { of baby's teeth, facial wipes, to } \\
\text { ward off snakes, facial, hair } \\
\text { grower }\end{array}$ & 0.25 & - & 0.795 & - \\
\hline
\end{tabular}

(-) - no reported incidence of the use of plant for treatment of the disease/ailment under the specific category

with a value of 0.84 and 0.833 respectively. Plants noted in these particular categories are lamibu, cabo negro (Cyathea contaminans (Wall. ex Hook.) Copel.), kamatesemala, tawa-tawa (Euphorbia hirta L.), and tino. Freshly collected roots of lamibu with its aromatic smell are inhaled by a person experiencing nausea, while the stem of cabo negro (Cyathea contaminans (Wall. ex Hook.) Copel.) is shaved, exposed to smoke and then topically applied to an epileptic person. For kamatesemala, shoot of flower is extracted, its juice applied externally on areas with German measles or areas showing redness.

People in the Obo community have a high consensus with regards to the plants they utilize for treating and preventing diseases. The healer plays a vital role in the community, serving as source of information in the treatment of various illnesses in 
the area. In fact, most young mothers sought advice from their community healer for their family's medical problems and thus become learned in medicinal plants. Sitio Lambila, where the study was conducted is a very remote area with no electricity and no constant supply of potable water. With these conditions, illnesses prevalent in the area are diarrhea and boils, which are associated with the lack of clean water supply (PGO-South Cotabato, 2010). According to the respondent, the community would be fortunate if once a month a barangay medical team could visit the area to provide very basic medical need. Thus, knowledge in plant use for its medicinal value proves to be essential in this remote community.

Respiratory diseases (0.4) showed the highest ICF, followed by gastrointestinal and internal parasite problems $(0.36)$ and in close third is the genito-urinary related problem $(0.33)$ in the T'boli community (Table 2). Mayana (Plectranthus scutellarioides (L.) R.Br.), Kyobog, Lagundi (Vitex negundo L). , and Aritis-aritis (Hyptis capitata Jacq).were used to treat cough. Mansanitas (Ziziphus jujuba Mill.), bayabas Psidium guajava L., tawa-tawa (Euphorbia hirta L.), mayana (Plectranthus scutellarioides (L.) R.Br.), Aritisaritis (Hyptis capitata Jacq), dila-dila, saging (Musa spp.), mama (Areca catechu L) were taken orally for gastric problem such as stomachache, diarrhea, and worm infestation. Lawaan (Shorea sp.), falsomangayan, carabao grass (Paspalum conjugatum P.J.Bergius), kokounbaya, kluonwaya, tawatawa (Euphorbia hirta L.), kuyoiwas, munot, fet (Eleusine indica (L.) Gaertn.) sampa-samplalukan (Phyllanthus niruri L.), kayu mlato were used to treat reproductive related problem such as bleeding after delivery and to facilitate easy child delivery.

The Tagakaolo community in Brgy. Conel, has a high consensus on the plants they utilized to treat various diseases. Treatment for bughat showed the highest ICF followed by medicine to prevent or reduce bleeding (0.880) (Table 2). However, their pharmacopeia is not species-rich as very few usable plants were recorded. Moreover, the types of ailments could be treated by the assemblage of medicinal plants are less diverse. It is worth noting though that this IP community constitute only a small number of households who migrated from Davao del Sur.

The use of traditional medicine is common in many parts of the world particularly among IPs whose connection to their natural environment is undeniably an essential part of their culture (Trotter and Logan, 1986). This idea holds true in the area as shown by the present assessment of Bla'an, T'boli, Tagakaolo and Obo tribes. The ethnobotanical knowledge and practices of the four IP communities stems from its long history of use, familiarity to the effects, availability and nearness of resources and spiritual connection. This traditional practice together with limited access and services to primary health care leads to its popularity. A high consensus among informants from the Obo community of Lake Sebu, South Cotabato was observed in contrast to the very low consensus of informants on the use of medicinal plants in Blaan community of Upper Labay, General Santos City, and T'boli community of Maitum, Sarangani. However, the consensus among the informants from the Tagakaolo community of Brgy Conel, General Santos City was high.

\section{Conclusion}

The four indigenous people's communities widely practice the use of medicinal plants in treating diseases and are therefore rich in ethnomedicinal knowledge. In this era of modern health care, the use of floral resources integrated into their culture and the inadequate basic health care in these communities are the reasons for the fortitude and even popularity of this practice. The persistence of the use of medicinal plants throughout the generation and the high consensus of users in the community may be used as an indication of their efficacy. Indeed, indigenous communities are repository of rich pharmacopeia and, when explored, serves as guide in the discovery of novel drugs.

\section{Acknowledgement}

The researchers are grateful to the Department of Agriculture-Bureau of Agricultural Research for the funding support; Mindanao State University General Santos City for allowing us to conduct this research; the Department of Environment and Natural Resources-Region 12 for the gratuitous permit enabling collection of sample plant specimens; the National Commission on Indigenous People (NCIP) of Region 12; and the Tribal Councils of Maitum, Lake Sebu and Brgy Upper Labay, General Santos City for granting permits to conduct this study. Special thanks also goes to $\mathrm{Mr}$. Renan Gubalane and Ms. Mia Betrice Pernia for the assistance in the data collection and photodocumentation. 


\section{References}

Azmir, J., Zaidul, I., Rahman, M., Sharif, K., Mohamed, A., Sahena, F., Jaharul M., Ghafoon K., Norulaini N., and Omar, A. (2013). Techniques for extraction of bioactive compounds from plant materials: A review. Journal of Food Engineering, 117(4), 426-436. doi:10.1016/ j.jfoodeng.2013.01.014

Brgy Upper Labay Profile Report. (2012) General Santos City: unpublished.

Capasso F, Gaginella T, Grandolini, G . (2003). Phytotherapy: A Quick Reference to Herbal Medicine. Berlin: Springer.

Castro, I. R. (2006). A Guide to Families of Common Flowering Plants in the Philippines. Diliman, Quezon City: The University of the Philippines Press.

Conservation International Philippines. Protected Areas and Wildlife Bureau. Haribon Foundation. (2007). Priority Sites for Conservation in the Philippines: Key Biodiversity Areas. Quezon City: Conservation International Philippines.

dela Cruz, P., and Gorospe-Ramos, A. (2006). Indigenous Health Knowledge Systems in the Philippines: A Literature Survey. Manila: Anvil Publishing Incorporated.

FDA. (2010). Home - Food and Drug Authority of the Philippines. Retrieved July 8, 2011, from http:// www.fda.gov.ph

Ferreira, F. S., Brito, S. V., Ribeiro, S. C., Saraiva, A. A., Almeida, W. O., \& Alves, R. R. (2009). Animal-based folk remedies sold in public markets in Crato and Juazeiro do Norte, Ceará, Brazil. BMC Complementary and Alternative Medicine, 9(1). doi:10.1186/1472-6882-9-17

Garcia, D., Domingues, M. V., \& Rodrigues, E. (2010). Ethnopharmacological survey among migrants living in the Southeast Atlantic Forest of Diadema, Sao Paulo, Brazil. Journal of Ethnobiology and Ethnomedicine, 6(1), 29. doi:10.1186/17464269-6-29

Gilani, A. H., \& Atta-ur-Rahman. (2005). Trends in ethnopharmacology. Journal of Ethnopharmacolo$g y$, 100(1-2), 43-49. doi:10.1016/j.jep.2005.06.001
Google (n.d.) [Map of South Cotabato Province, General Santos City and Sarangani Province]. Retrieved January 25, 2017, from www.Googleearth.com.

Heinrich, M., \& Gibbons, S. (2001). Ethnopharmacology in drug discovery: an analysis of its role and potential contribution. Journal of Pharmacy and Pharmacology, 53(4), 425-432. doi:10.1211/0022357011775712.

Kumar, B., Vijayakumar, M., Govindarajan, R., \& Pushpangadan, P. (2007). Ethnopharmacological approaches to wound healing-Exploring medicinal plants of India. Journal of Ethnopharmacology, 114(2), 103-113. doi:10.1016/ j.jep.2007.08.010.

Leffers, A. (2003). Gemsbok Bean \& Kalahari Truffle: Traditional Plant Use by Jul'hoansi in North-Eastern Namibia. Windhoek: Gamsberg Macmillan.

Mendoza, R. (2009). Is It Really Medicine? The Traditional and Alternative Medicine Act and Informal Health Economy in the Philippines. Asia Pacific Journal of Public Health, 21(3), 333-345. doi:10.1177/1010539509336570.

Nishijima, C. M., Rodrigues, C. M., Silva, M. A., Lopes-Ferreira, M., Vilegas, W., \& HirumaLima, C. A. (2009). Anti-hemorrhagic Activity of Four Brazilian Vegetable Species Against Bothrops jararaca Venom. Molecules, 14(3), 10721080. doi:10.3390/molecules 14031072 .

Palaganas, E. C., \& University of the Philippines. (2001). Mainstreaming Indigenous Health Knowledge and Practices. Diliman, Quezon City: University of the Philippines, Center for Integrative and Development Studies.

Panghal, M., Arya, V., Yadav, S., Kumar, S., \& Yadav, J. (2010). Indigenous knowledge of medicinal plants used by Saperas community of Khetawas, Jhajjar District, Haryana, India. Journal of Ethnobiology and Ethnomedicine, 6(1), 4. doi:10.1186/1746-4269-6-4.

Peter, P.B, Barcelone, J.F., \& Nickerny, D.L., (2011). Co's Digital Flora of the Philippines. Retrieved January 2012 from www.philippineplants.org 
Peralta, J. (2003). Glimpses: Peoples of the Philippines. Manila: Anvil Publishing Incorporated.

Pfeiffer, J. M., \& Butz, R. J. (2005). Assessing cultural and ecological variation in ethnobiological research: the importance of gender. Journal of Ethnobiology, 25(2), 240-278. doi:10.2993/0278-0771 (2005)25[240:acaevi]2.0.co;2.

Pieters, L., \& Vlietinck, A. J. (2005). Bioguided isolation of pharmacologically active plant components, still a valuable strategy for the finding of new lead compounds? Journal of Ethnopharmacology, $\quad$ 100(1-2), 57-60. doi:10.1016/ j.jep.2005.05.029

PGO-South Cotabato Information Office. (2013). General Information. Retrieved November 16, 2016, from http://www.southcotabato.gov.ph

PGO-South Cotabato Information Office. (2010). History and people of South Cotabato. Retrieved July 15, 2011, from http:// www.southcotabato.gov.ph

Philippine Statistics Authority. (2012). Welcome to CountrySTAT Philippines. Retrieved August 12, 2012, from http://countrystat.psa.gov.ph PPDO. (2010). Province of Sultan Kudarat - Experience Diversity and Beauty. Retrieved August 15, 2017, from http://sultankudaratprovince.gov.ph

Sarangani Provincial Information Unit. (2010). Profile of Sarangani Province, the history and people of Sarangani Province. Retrieved January 2015 from http://www.sarangani.gov.ph

Sher, H., Alyemeni, M., and Faridullah (2010). Cultivation and Domestication study of High-Value Medicinal Plant Species (its economic potential and linkages with commercialization). African Journal of Agricultural Research, Vol. 5 (18): 2462 -2470 .

Sivaperumal, R., Ramya, S., Veera Ravi, A., Rajasekaran, C., Jayakumararaj, R. (2010). Ethnopharmacological Studies On The Medicinal Plants Used By Tribal Inhabitants Of Kottur Hills, Dharmapuri, Tamilnadu, India. Environment and We: An International Journal of Science and Technology. 5: 57 -64 .

Tabuti J.R.S, Kukunda C.B, Kaweesi D., Kasilo O.M.J. (2012). Herbal medicine use in the district of Nakapiripirit, Pallisa, Kanungu and Mukono in Uganda. Journal of Ethnobiology Ethnomedicine. 2012:8-35

The Plant List (2013). Version 1.1. Published on the Internet, Accessed November 2017 from http:// www.theplantlist.org/

Trotter R., \& Logan M. (1986). Informant consensus: A new approach for identifying potentially effective medicinal plants. In Plants in Indigenous Medicine and Diet: Biobehavioral Approaches. New York: Regrave Publishers.

Union internationale pour la conservation de la nature et de ses ressources. Organisation mondiale de la sante? Fonds mondial pour la nature. (1993). Guidelines on the conservation of medicinal plants. Gland: The International Union for Conservation of Nature and Natural Resources.

Wikipedia. (2018, March 1). Soccsksargen map. Retrieved February 2, 2018, from https:// en.wikipedia.org/wiki/SOCCSKSARGEN 